ARTICLE

DOI: $10.1038 /$ s41467-017-01322-9

\title{
Quantitative microscopy of the Drosophila ovary shows multiple niche signals specify progenitor cell fate
}

Wei Dai ${ }^{1}$, Amy Peterson ${ }^{1}$, Thomas Kenney ${ }^{1}$, Haley Burrous ${ }^{1} \&$ Denise J. Montell (i] ${ }^{1}$

Adult stem cells commonly give rise to transit-amplifying progenitors, whose progeny differentiate into distinct cell types. It is unclear if stem cell niche signals coordinate fate decisions within the progenitor pool. Here we use quantitative analysis of $\mathrm{Wnt}, \mathrm{Hh}$, and Notch signalling reporters and the cell fate markers Eyes Absent (Eya) and Castor (Cas) to study the effects of hyper-activation and loss of niche signals on progenitor development in the Drosophila ovary. Follicle stem cell (FSC) progeny adopt distinct polar, stalk, and main body cell fates. We show that Wnt signalling transiently inhibits expression of the main body cell fate determinant Eya, and Wnt hyperactivity strongly biases cells towards polar and stalk fates. Hh signalling independently controls the proliferation to differentiation transition. Notch is permissive but not instructive for differentiation of multiple cell types. These findings reveal that multiple niche signals coordinate cell fates and differentiation of progenitor cells. 
A dult stem cells are important for tissue homoeostasis and regeneration due to their ability to both self-renew and generate multiple types of differentiated daughters. Adult stem cells are located in a niche that provides the proper microenvironment to maintain "stemness"1, 2. The progeny of stem cells that move away from the niche generally go through a precursor cell (or progenitor cell, transit-amplifying cell) stage before they differentiate ${ }^{3,4}$. However, it is unclear whether the precursor state is simply a loss of stemness due to displacement from niche signals, or whether secreted niche factors might act as graded morphogens that establish distinct cell fates at different concentrations and distances from the niche.

The Drosophila ovary is an appealing model for studying adult stem cells ${ }^{5}$. Each ovary contains 16-20 ovarioles, which are chains of egg chambers in increasing stages of maturity ${ }^{6}$ (Fig. 1a). Development begins in the germarium, which is located at the anterior tip of the ovariole. The anterior half of the germarium, region 1, contains germline stem cells and their progeny, which continue dividing to produce 16-cell cysts. Somatic escort cells surround the developing cysts as they progress to region $2 \mathrm{a}$. The FSCs are located at the region $2 \mathrm{a} / 2 \mathrm{~b}$ boundary ${ }^{7}$, where cysts exchange their escort cell covering for the FSC daughters. The posterior half of the germarium contains flattened cysts in region $2 \mathrm{~b}$, followed by rounded region 3 cysts. Follicle precursor cells associate with region $2 \mathrm{~b}$ and region 3 cysts, and their progeny adopt distinct polar, stalk, and main body cell fates, which serve different functions. However, the molecular mechanisms that govern these earliest cell fate decisions are mostly unknown and most precursors in region $2 \mathrm{~b}$ and region 3 do not yet express mature cell fate markers ${ }^{8-10}$.

Several signalling pathways have been implicated in regulating follicle precursor cell fate specification and differentiation. Notch signalling is required for polar cell specification ${ }^{9}$ and is present in mature polar cells at high levels in region $3 /$ stage $1^{11}$. Earlier Notch activity at the region $2 \mathrm{a} / 2 \mathrm{~b}$ boundary is required for one FSC daughter to migrate laterally across the germarium, while

a

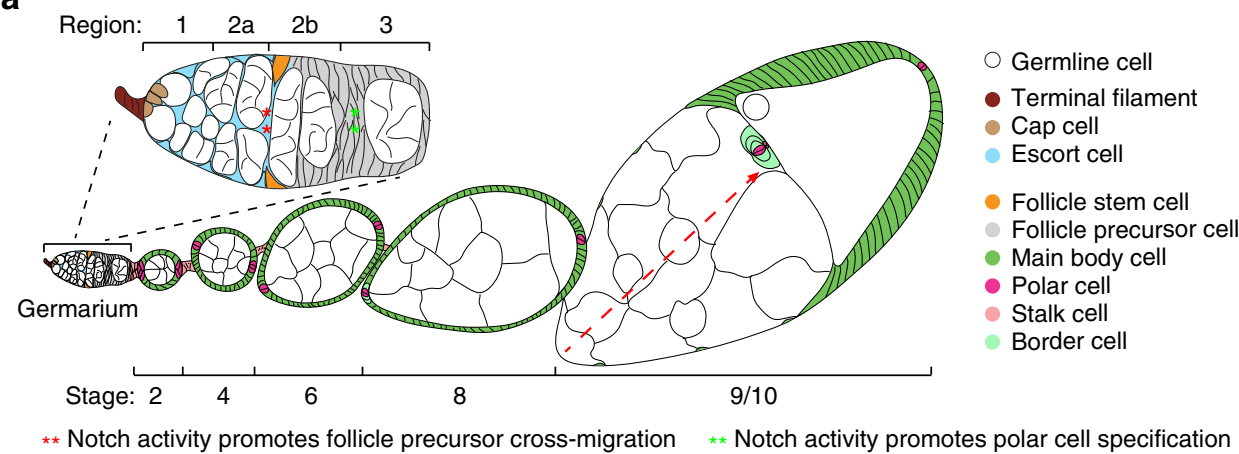

b

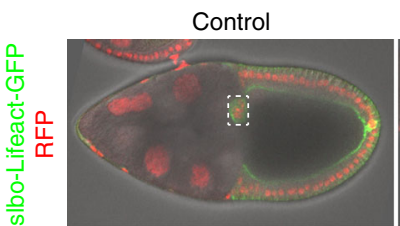

d

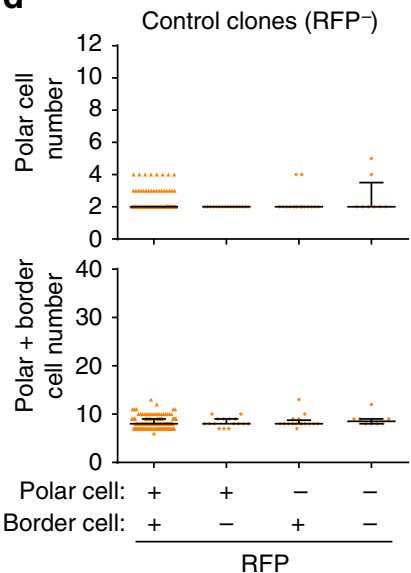

Axn-
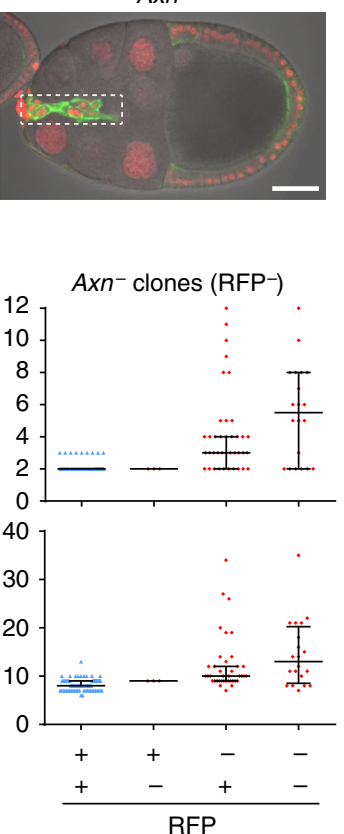

C

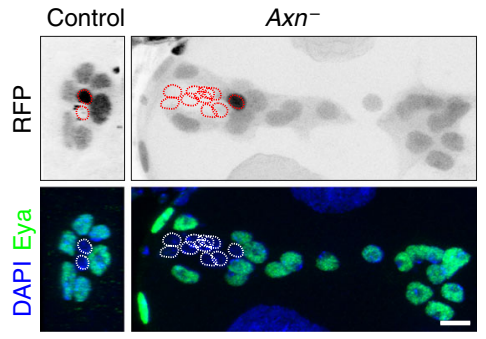

e Control ptc-

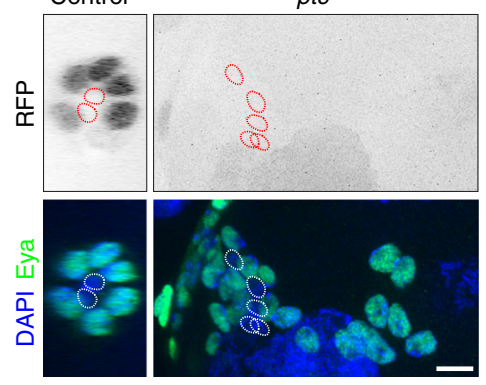

Fig. 1 Axn mutant clones cause supernumerary polar cells. a Drawing of a Drosophila ovariole in the sagittal view. Dashed arrow indicates the border cell migration path. b Sagittal view of stage 10 egg chambers with control (left panel) or FRT82B, Axn ${ }^{1511}$ mosaic (right panel) border cell clusters (dashed boxes). Scale bar, $50 \mu \mathrm{m}$. c 3D projection view of border cell clusters containing FRT82B control or FRT82B, Axn ${ }^{1511}$ mosaic clones. Homozygous mutant cells are RFP_negative (RFP ${ }^{-}$). Polar cells are identified by absence of Eya expression (dotted circles). Scale bar, $10 \mu \mathrm{m}$. $\mathbf{d}$ Quantification of all border cell clusters in stage $9 / 10$ egg chambers, regardless of whether they have clones or not, in FRT82B control or FRT82B, Axn ${ }^{1511}$, 4-5 days after clone induction. Data from $n=284$ egg chambers for control, 222 for $\mathrm{Axn}^{-}$. Each dot represents one border cell cluster. Lines show the median with interquartile range. e Border cell cluster in FRT42D control or FRT42D, ptc ${ }^{S 2}$ mutant clones. Homozygous mutant cells are RFP- ${ }^{-}$. Polar cells are Eya ${ }^{-}$(dotted circles). Scale bar, $10 \mu \mathrm{m}$ 
a

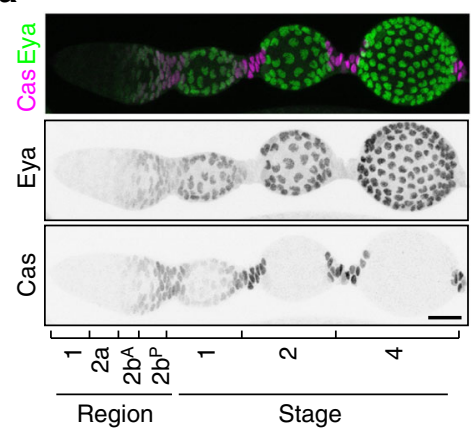

b

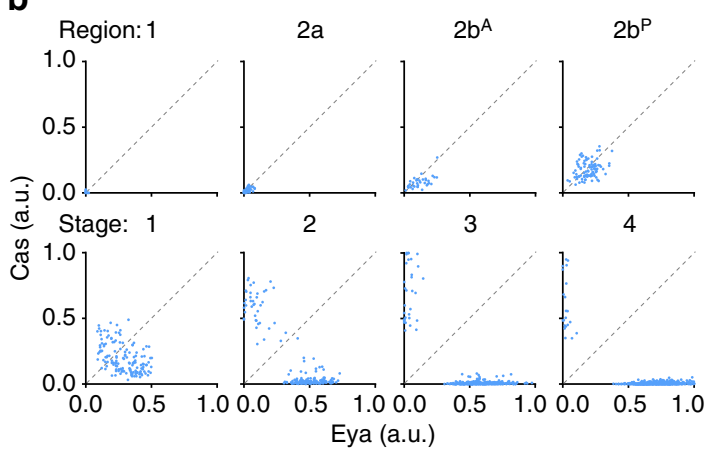

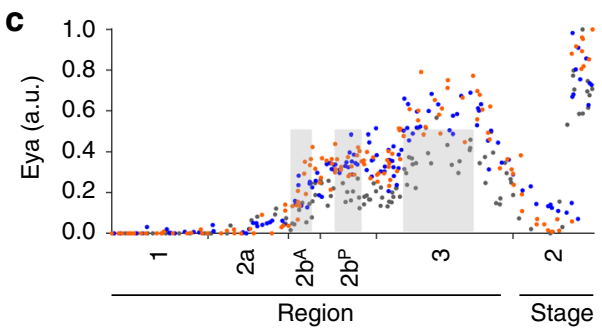

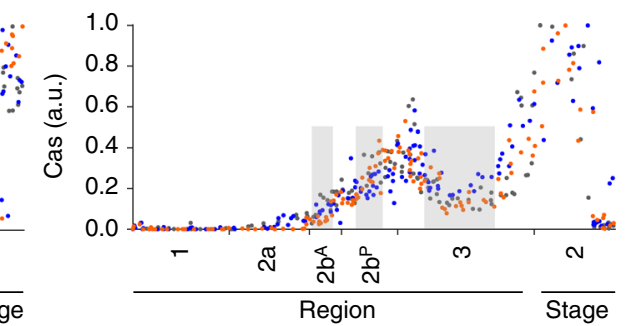

d

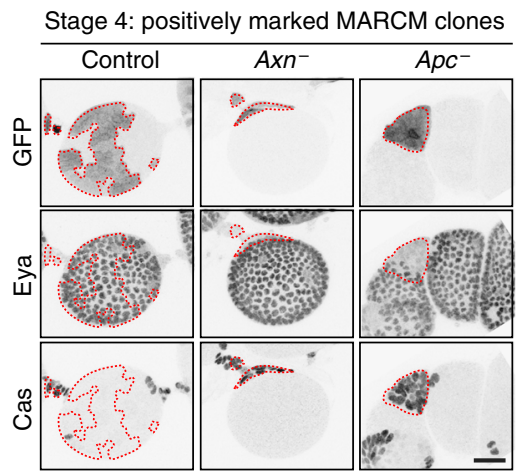

e Stage 4: negatively marked mosaic clones

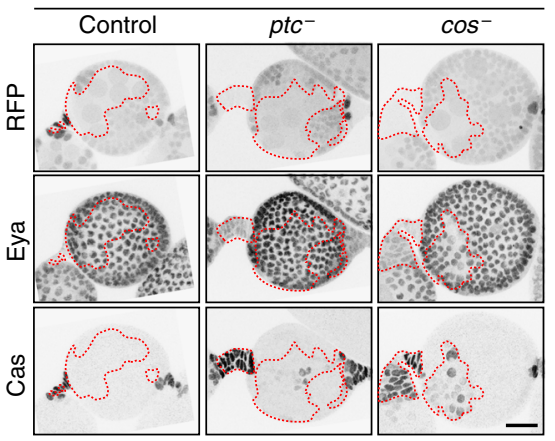

$\mathbf{f}$

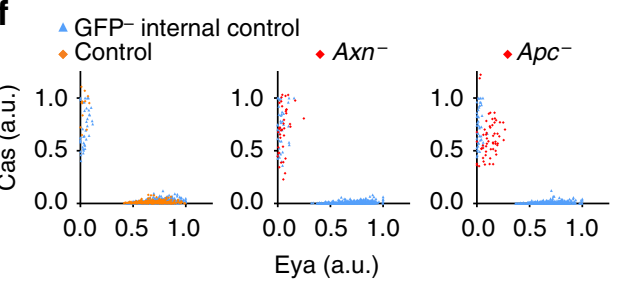

g $\triangle \mathrm{RFP}+$ internal control

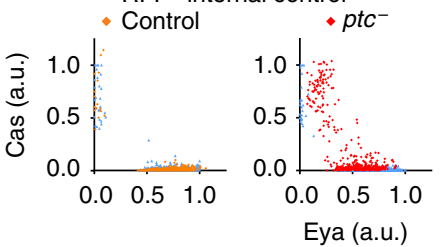

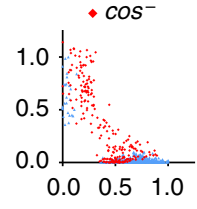

Fig. 2 Differential effects of Wnt and Hh hyper-activation on follicle cell differentiation. a 3D projection view of one half of a wild type ovariole from the germarium to stage 4, stained with Eya (green) and Cas (magenta) antibodies. Individual channels are shown in black and white. $\mathbf{b}$ Quantification of Eya and Cas fluorescence intensity in all somatic cells in germarium regions 1-3 and in stage 1-4 egg chambers. Data from $n=2122$ cells from 3 ovarioles per stage. Each dot represents one cell. Data were normalized to maximum Eya or Cas intensity per ovariole. c Quantification of Eya and Cas intensity in all somatic cells in germarium until anterior of stage 2 along the anterior-posterior axis. Data from $n=399$ cells from three ovarioles. Grey bars indicate position of the DNA from the germline cysts. Different colours represent different ovarioles. Data were normalized to maximum Eya and Cas intensity per sample. d Stage 4 egg chambers with FRT82B control mosaic FSC clones compared to FRT82B, Axn ${ }^{1511}$, or FRT82B, Apc $2^{910}$, Apc ${ }^{\mathrm{Q} 8}$. Homozygous mutant cells in the main body and anterior polar/stalk regions are outlined ( $\mathrm{GFP}^{+}$, dashed lines). Eya ${ }^{+} \mathrm{GFP}^{-}$cells appear in the outlined $A p c^{-}$clone due to $Z$ stack projection. e Stage 4 egg chambers with FRT42D control, FRT42D, ptc ${ }^{\mathrm{S} 2}$ or FRT42D, $\cos ^{\mathrm{H} 29}$ mosaic FSC clones. Homozygous mutant cells are RFP (dashed lines). $\mathbf{f}, \mathbf{g}$ Quantification of Eya and Cas fluorescence intensity in mosaic FSC clones. Data from $n=1222-1446$ cells from 4 stage 4 egg chambers per genotype. Data were normalized to maximum Eya or Cas intensity in internal control cells per egg chamber. Scale bars, $20 \mu \mathrm{m}$. a.u., arbitrary unit

other daughters move posteriorly ${ }^{8}$. However, Notch activity is not sufficient to induce ectopic polar cells in the main body region ${ }^{10,12}$, suggesting that additional factors control polar cell fate.

Escort cells form the FSC niche ${ }^{2,13,14}$. Niche factors important for FSC maintenance include Wnt, Hh, epidermal growth factor (EGF), and bone morphogenetic protein, which are crucial in many adult stem cell niches ${ }^{13}, 15-20$. Hyper-activation of Wnt or
Hh signalling causes defects in follicle cell differentiation ${ }^{15,21}$, but the origins of these phenotypes are not understood and it remains unclear whether these niche signals normally regulate progenitor cell fate or differentiation.

In a forward genetic screen for mutations that disrupt cell fates in the ovary, we identified a mutant allele of Axin $(A x n)$, a negative regulator in the Wnt pathway. The phenotype resembled that caused by mutations in patched (ptc) or costal (cos), two 
a

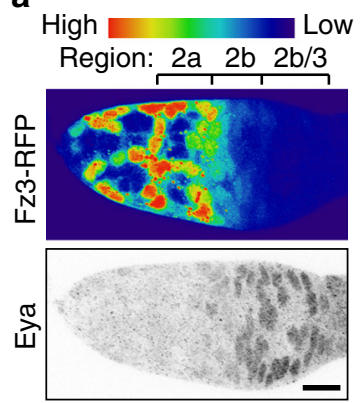

e

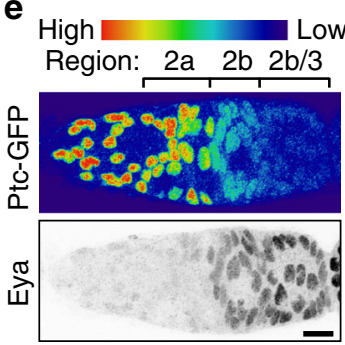

b

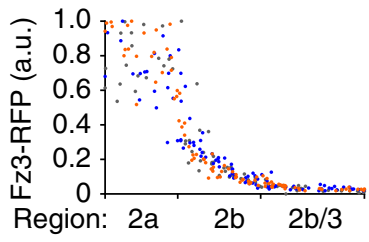

f

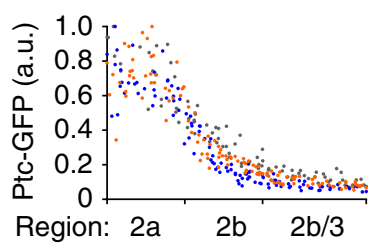

C

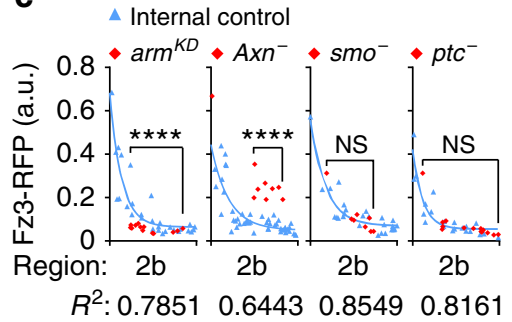

g

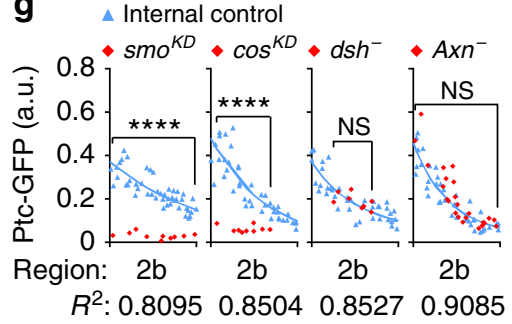

d

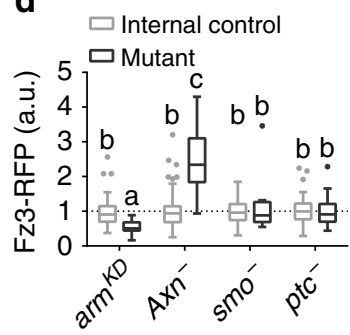

h

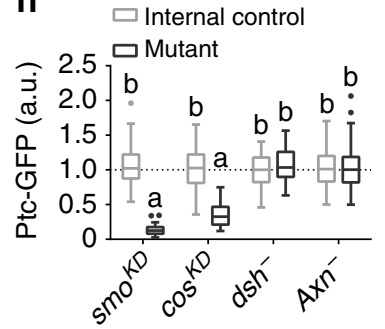

Fig. 3 Independent actions of Wnt and Hh in the germarium. a 3D projection view of one half of a germarium expressing the Wnt activity reporter fz3-RFP and stained for Eya. RFP intensity is displayed using the "Physics" lookup table. b Quantification of Wnt reporter intensity in all somatic cells from region 2-3 along the anterior-posterior axis. Data from $n=276$ cells from 3 germaria. Each dot represents one cell. The three colours represent the three different germaria. c Wnt reporter activity in germaria with armRNAi, $A x n^{S 044230}$, smo ${ }^{D 16}$, or ptc ${ }^{S 2}$ mutant clones (red diamonds) compared to control cells in the same germarium (blue triangles). $R^{2}$ quantifies goodness of fit of nonlinear regression. $\mathbf{d}$ Quantification of Wnt reporter activity in germaria region $2 \mathrm{~b}$ with mosaic clones. Data (median with interquartile range) from $n=16-103$ cells from 3 germaria per genotype. Data were normalized to the predicted value on the one phase decay curve fitted on the internal control cells. $P<0.01$. e A germarium expressing the Hh activity reporter ptc-GFP. $\mathbf{f}$ Quantification of Hh reporter intensity in all somatic cells from regions 2 to 3 along the anterior-posterior axis. Data from $n=314$ cells from 3 germaria. $\mathbf{g}$ Hh reporter activity in germaria with smoRNAi, $\cos R N A i, d s h^{3}$, or $A x n^{S 044230}$ mutant clones. h Quantification of $\mathrm{Hh}$ reporter activity in germarium region $2 \mathrm{~b}$ with mosaic clones. Data (median with interquartile range) from $n=18-105$ cells from 3 germaria per genotype. $P<0.0001$. Scale bars, $10 \mu \mathrm{m}$. ${ }^{\star \star} P<0.01$; ${ }^{\star \star \star \star} P<0.0001$ (Mann-Whitney test). Samples labelled with different letters are significantly different (Kruskal-Wallis with Dunn's test)

negative regulators of Hh signalling. However, when we traced both defects back to the earliest steps of follicle cell specification, we found differences. We developed quantitative analyses of the differentiation markers, Eya and Cas, as well as Wnt, $\mathrm{Hh}$ and Notch signalling reporters, which revealed independent roles for these three pathways. We found that the Wnt and Hh responses exhibited distinct signal patterns in the germarium. Wnt signalling transiently suppressed the main body cell fate factor Eya, whereas Hh signalling delayed differentiation of all follicle cell types. Loss of negative regulators caused more severe phenotypes than loss of positive regulators. Both loss and gain of function of the two pathways produced additive phenotypic effects. Notch knockdown caused multiple cell differentiation defects, but constitutively active Notch was not instructive for any particular cell fate. We conclude that combinatorial signalling produces the appropriate spatial patterning of cell types and temporal patterning of differentiation.

\section{Results}

Distinct effects of Wnt and Hh hyper-activation. At stage 8 of oogenesis, anterior polar cells specify neighbouring epithelial follicle cells as motile border cells, and together they migrate as a cluster during stage 9 (Fig. 1a). In a forward genetic screen of EMS-induced mutations that cause border cell defects in mosaic clones $^{22}$, we identified a line that produced abnormally large border cell clusters. Compared to control clusters, which are usually composed of 5-7 migratory cells surrounding two polar cells, clusters containing mutant cells showed as many as 6-12 polar cells and 14-35 total cells per cluster (Fig. 1b-d). The phenotype was autonomous to the polar cells, as supernumerary polar cells and over-sized border cell clusters were only observed when polar cells were homozygous for the mutation (Fig. 1d). The supernumerary polar cell phenotype resembled those previously reported for $e y a^{23}, \cos ^{24}$, and ptc (Fig. $1 \mathrm{e}^{21}$ ). We mapped the new mutation to genomic location 99D3 (Supplementary Fig. 1a-c), which contains the Axn gene. Axn allele S044230 produced a similar phenotype (Supplementary Fig. 2) and failed to complement the new mutation for lethality. We therefore named the new allele $A x n^{1511}$. This supernumerary polar cell phenotype was somewhat surprising, as it had not previously been reported for the Axn gene.

In addition to supernumerary polar cells, $A x n^{-}$and $p t c^{-}$clones showed abnormal stalks, consistent with previous reports ${ }^{15}, 25$. Polar and stalk cell specification occurs early in ovarian development, and these cells stop dividing soon after they exit the germarium ${ }^{7}, 26$. In the ovary, hyperactive Wnt or $\mathrm{Hh}$ signalling affects differentiation ${ }^{15}, 21$. To ask what aspects of differentiation were affected by Wnt or Hh hyper-activation, we used two complementary markers, Eya ${ }^{23}$ and Cas $^{27}$ (Fig. 2a). To be comprehensive, we performed $3 \mathrm{D}$ reconstructions of ovarioles and quantified the levels of both Eya and Cas in every somatic cell (Fig. 2b; Supplementary Fig. 3; Supplementary Movie 1). We found barely detectable levels of either protein in regions 1 and $2 \mathrm{a}$ escort cells. Low levels of both Eya and Cas were present in FSCs which increased in their daughters in anterior and posterior region $2 \mathrm{~b}\left(2 \mathrm{~b}^{\mathrm{A}}\right.$ and $\left.2 \mathrm{~b}^{\mathrm{P}}\right)$. Eya and Cas showed differential expression in region 3/stage 1 , such that some cells expressed higher levels of one or the other (indicated by divergence from the diagonal in the graphs in Fig. 2b). As development proceeded through stages 1-4, the levels of Eya and Cas diverged more and more. By stage 4, Eya and Cas became completely distinct markers for main body $\left(\mathrm{Eya}^{+} \mathrm{Cas}^{-}\right)$versus polar or stalk (Eya ${ }^{-}$ 
$\mathrm{Cas}^{+}$) fates (Fig. 2a, b). Further detailed examination revealed that the Eya level increased precipitously within region $2 \mathrm{~b}^{\mathrm{A}}$ along the anterior-posterior axis, while the Cas level increased more gradually throughout the $2 \mathrm{~b}^{\mathrm{A}}$ and $2 \mathrm{~b}^{\mathrm{P}}$ regions (Fig. 2c; Supplementary Fig. 4). The combination of Eya and Cas distinguished cells in region $2 \mathrm{~b}$, where both markers are low but increasing from those in region 3/stage 1, which began to diverge into Eya ${ }^{\text {low }}$ Cas ${ }^{\text {high }}$ and Eya ${ }^{\text {high }}$ Cas ${ }^{\text {low }}$ cells though many cells still express both to some degree. Thus, Eya and Cas are excellent markers for studying the earliest cell fates in the ovary.
To characterize the defects caused by hyperactive Wnt or Hh signalling more precisely, we made FSC clones and stained them for Eya and Cas (Fig. 2d, e). Hyperactive Wnt signalling, caused by loss of the destruction complex component $A x n^{-}$or adenomatous polyposis coli $\left(A p c^{-}\right)$produced many egg chambers containing only Eya ${ }^{-} \mathrm{Cas}^{+}$clones, in contrast to controls in which $\mathrm{Eya}^{+}$cells were frequent (Fig. 2d, f). By contrast, FSC clones with hyperactive $\mathrm{Hh}$ signalling caused by loss of the negative regulators $p t c^{-}$or $\cos ^{-}$were not biased towards polar/stalk fates or terminal positions. They instead produced many $\mathrm{Eya}^{+} \mathrm{Cas}^{+}$
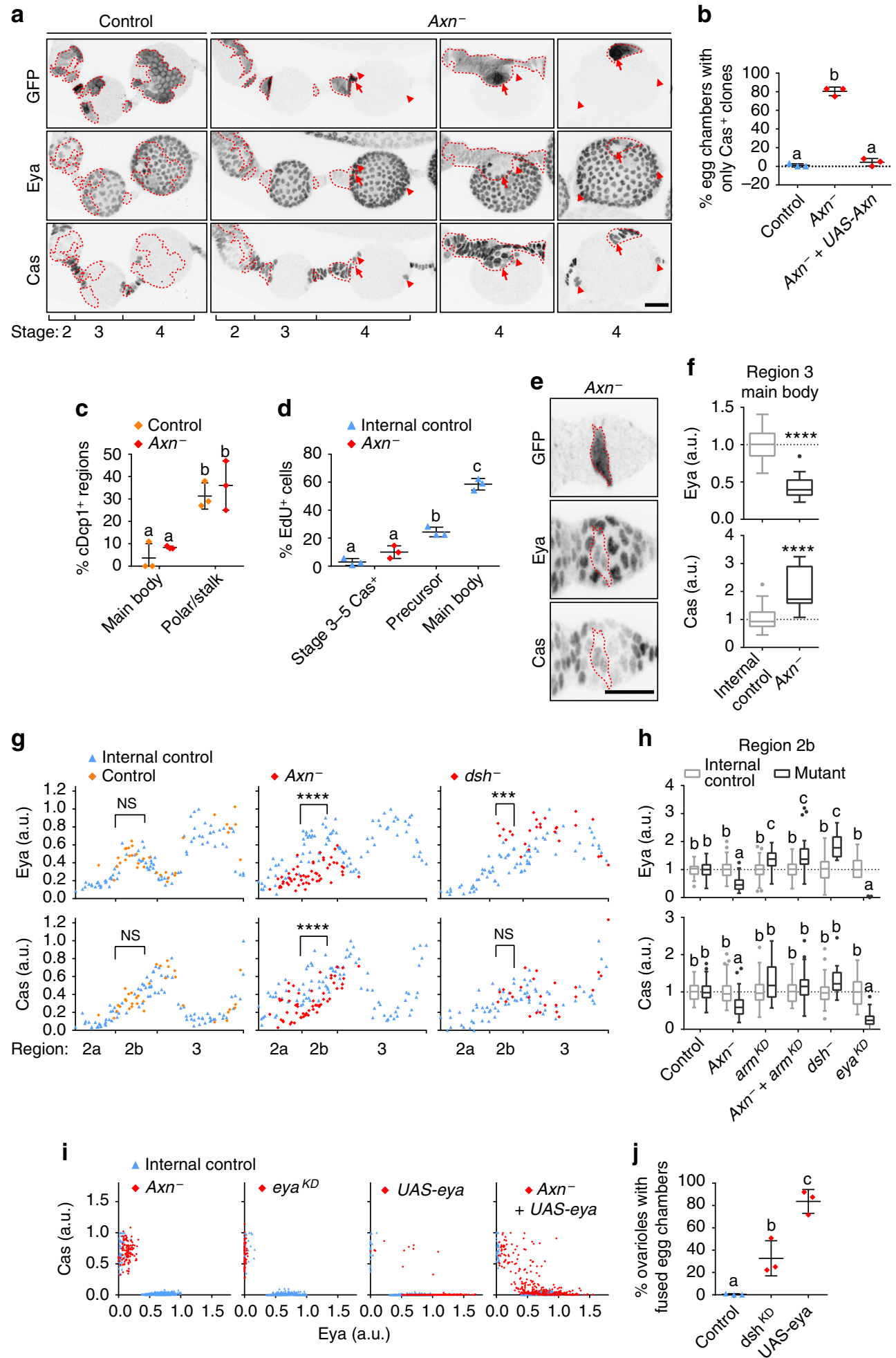
cells in stage 4 that resembled control cells in stages 1 and 2. Eya ${ }^{+}$ $\mathrm{Cas}^{+}$cells were virtually never observed in controls in stage 4 (Fig. 2b, e, g). These results suggested distinct responses to Wnt or Hh hyper-activation.

Wnt and Hh act independently in the germarium. Wnt and $\mathrm{Hh}$ signalling positively regulate one another in some settings ${ }^{28}$, while they antagonize ${ }^{29}, 30$ or play independent roles ${ }^{31}$ in other cases. In the ovary, Wnt and Hh are stem cell niche factors produced in cap cells and escort cells $13,19,20,32$. To understand their relationship, we examined $\mathrm{Wnt}$ and $\mathrm{Hh}$ activity patterns. We used frizzled $3(f z 3)-R F P^{33}, 34$, which was previously verified as the best available reporter for Wnt signalling activity in the germarium as it is most consistent with the expression patterns of Wnt ligands ${ }^{34}$. Fz3-RFP was highly expressed in regions $1-2 \mathrm{a}$ and showed a graded pattern in region $2 b$ (Fig. $3 a, b)$. To verify the fidelity of the reporter, we performed mosaic analysis. Reducing expression of the positive regulator of Wnt signalling $\beta$-catenin (in Drosophila Armadillo (Arm)) by RNAi reduced the Fz3-RFP signal and Fz3-RFP increased in $A x n^{-}$clones, demonstrating that it is indeed responsive to Wnt signalling (Fig. 3c, d; Supplementary Fig. 5a). The level of Fz3-RFP expression present in $A x n^{-}$cells in region $2 b^{\mathrm{P}}$ was about $\sim 30 \%$ of the maximum endogenous level, found in escort cells, and $\sim 75 \%$ of the level normally found in region $2 \mathrm{~b}^{\mathrm{A}}$, thus well within the physiological range.

To decipher the relationship between Wnt and Hh signalling, we examined the pattern of $f z 3-R F P$ in $s m o^{-}$or $p t c^{-}$clones. Changing $\mathrm{Hh}$ signalling had no detectable effect on the Wnt reporter in region $2 b$ (Fig. $3 c, d$ ). ptc-GFP is a reporter for Hh signalling $^{13}$ and shows a pattern similar to $f z 3-R F P$ (Fig. 3e, f), though the signal extends more posteriorly. The Ptc-GFP signal was reduced when knocking down smoothened (smo), a positive regulator in the Hh pathway (Fig. 3g, h; Supplementary Fig. 5b). Unexpectedly, cosRNAi also caused a reduction of Ptc-GFP signal in region $2 \mathrm{~b}$ (Fig. 3g, h; Supplementary Fig. 5c), while in later stages the signal increased as expected for loss of a negative regulator (Supplementary Fig. 6). The pattern of Ptc-GFP in dishevelled ( $d s h$, a positive regulator in the Wnt pathway) or $A x n$ mutant clones was not measurably different from control clones. (Fig. 3g, h). Thus, Wnt and Hh appear to function independently in the ovary.

Wnt signalling inhibits expression of the main body factor Eya. $A x n^{-}$FSC clones frequently gave rise exclusively to $\mathrm{Cas}^{+}$cells (Fig. 4a, b). $A x n^{-}$clones appear in the normal polar/stalk region, or as small clones within the main body forming ectopic polar and stalk cells, or as large clones that form a continuous stalk with a single polar cell cluster, causing the egg chamber to appear to bud from the side. Clones generated at a later stage, however, did not show this cell fate bias (Supplementary Fig. $7^{15}$ ), suggesting a narrow developmental time window for Wnt signalling to affect cell fate. To understand how hyper-activation of Wnt biased cells towards $\mathrm{Cas}^{+}$polar/stalk-like fates, we considered a few possibilities. The $A x n^{-} \mathrm{Eya}^{+}$main body precursors may not survive, or $\mathrm{Cas}^{+}$polar/stalk-like cells may proliferate more. Alternatively, or in addition, more cells may adopt a polar/stalk-like fate than a main body fate.

During early stages of oogenesis, apoptosis was common in polar and stalk cells but rare in main body cells (Fig. $4 \mathrm{c}^{35}$ ). $A x n^{-}$ clones in region 3-stage 2 main body regions did not show a detectable increase in apoptosis (Fig. 4c). The mitotic marker 5-ethynyl-2'-deoxyuridine (EdU) showed a slight increase in $\mathrm{Axn}^{-}$ $\mathrm{Cas}^{+}$cells that did not rise to statistical significance (Fig. 4d) compared to control Cas ${ }^{+}$cells in stages 3-5, suggesting that these polar/stalk-like cells did not proliferate more, consistent with previous observations ${ }^{15}$. Strikingly, we found a decrease of Eya and increase of Cas in the main body region in $\mathrm{Axn}^{-}$cells as early as region 3, suggesting a shift in cell fate (Fig. 4e, f). Such small clones in the main body region likely gave rise to ectopic Eya ${ }^{-}$ $\mathrm{Cas}^{+}$clones observed in stage 4 egg chambers, whereas large main body clones likely developed into continuous stalks with a single polar cell cluster, causing the egg chamber to appear to bud from the side (Fig. 4a).

To understand how Wnt signalling affects follicle precursor cells, we quantified changes in Eya and Cas levels in germarium region $2 b$ (Supplementary Movies 2-4). Eya was significantly reduced in the $A x n^{-}$clones (Fig. $4 \mathrm{~g}, \mathrm{~h}$ ), which activated Wnt signalling in region $2 b^{\mathrm{P}}$ to the level normally found in region $2 \mathrm{~b}^{\mathrm{A}}$ (Fig. 3c). Conversely, $d s h^{-}$cells showed significantly increased Eya in region $2 \mathrm{~b}$ to a level closer to region 3 main body cells (Fig. $4 \mathrm{~g}, \mathrm{~h}$ ). The Eya intensity in region $2 \mathrm{~b} d s h^{-}$cells was on average 1.83-fold compared to control cells in region $2 \mathrm{~b}$ and 0.84 fold compared to control cell in region 3, suggesting that Wnt signalling normally functions to inhibit Eya expression. Reducing Wnt signalling by armRNAi also resulted in increased Eya, relative to control (Fig. $4 \mathrm{~h}$ ). In double $A x n^{-} / \mathrm{armRNA} i$ cells, arm was epistatic to $A x n$ as expected (Fig. 4h). Cas was also reduced in $A x n^{-}$cells in region $2 \mathrm{~b}$, but less so than Eya. Cas was similarly reduced in eya knockdown cells in this region (Fig. $4 \mathrm{~g}, \mathrm{~h}$ ), so the reduction of Cas in $\mathrm{Axn}^{-}$could be a secondary consequence of Eya reduction.

Eya is a potent determinant of polar and stalk cell fates, and knocking eya down in mosaic clones caused all mutant cells to become $\mathrm{Eya}^{-} \mathrm{Cas}^{+}$in stage 4 egg chambers, which phenocopied $A x n^{-}$(Fig. 4i). To test how important the reduction of Eya was for the fate change in $\mathrm{Axn}^{-}$cells, we expressed UAS-eya in $\mathrm{Axn}^{-}$ clones. Eya expression restored main body cell fate to many cells (Fig. 4i; Supplementary Fig. 8a, b). Therefore, Eya is a key target

Fig. 4 Wnt signalling inhibits expression of the main body cell fate factor Eya. a 3D projection view of one half of ovarioles with FRT82B control or Axn $^{\mathrm{S} 044230}$ mutant (GFP ${ }^{+}$, dashed lines) FSC clones. Arrowheads point to control polar cells in stage 4 of Axn ${ }^{-}$, arrows point to the mutant polar cells. Eya ${ }^{+}$ $\mathrm{GFP}^{-}$cells appear in the outlined stage 4 Axn ${ }^{-}$clones due to Z stack projection. $\mathbf{b}$ Stage 3-5 egg chambers with Cas ${ }^{+}$only clones. Data (mean \pm s.d.) from 3 experiments, 77-89 egg chambers per genotype. $P<0.0001$. c Main body or polar/stalk regions with cells expressing cDcp1 in region 3-stage 2 FSC clones. Data (mean \pm s.d.) from 3 experiments, $24-36$ egg chambers. $P<0.05$. d EdU incorporation in Axn ${ }^{S 044230}$ heterozygous control or homozygous mutant. Data (mean \pm s.d. from three experiments, 446-812 stage 3-5 Cas ${ }^{+}$cells, 465 germarium region 2 b control cells, 1503 stage 3 main body control cells, $P<0.01$. e 3D projection view of one half of a stage 1 egg chamber with Axn ${ }^{S 044230}$ FSC clones. $\mathbf{f}$ Eya and Cas intensity in region 3 main body cells. Data (median with interquartile range) from three egg chambers, 17-59 cells. Data were normalized to average Eya or Cas in internal control cells. $\mathbf{g}$ Eya and Cas intensity in germaria with FRT82B control (orange diamonds), $\mathrm{Axn}^{\mathrm{SO} 44230}$, or $d s \mathrm{~h}^{3}$ mutant clones (red diamonds) compared to control cells in the same germarium (blue triangles). $\mathbf{h}$ Eya and Cas intensity in germarium region $2 \mathrm{~b}$. Data (median with interquartile range) from $33-100$ cells from 4 germaria per genotype. $P<0.01$. i Eya and Cas intensity in stage 4 follicle cells. 1365-1434 cells from 4 stage 4 egg chambers per genotype. j c306-Gal4-driven dsh knockdown or UAS-eya overexpression in follicle precursor cells. Data (mean \pm s.d.) from 3 experiments, $213-252$ ovarioles. $P<0.05$. Scale bars, $20 \mu \mathrm{m} .{ }^{\star \star \star} P<0.001 ;{ }^{\star \star \star} P<0.0001$ (Mann-Whitney test). Samples labelled with different letters are significantly different using ANOVA with Turkey's test b-d, j, or Kruskal-Wallis with Dunn's test $\mathbf{h}$ 
of hyperactive Wnt signalling. Expression of Eya in $A x n^{-}$clones also produced $\mathrm{Eya}^{+} \mathrm{Cas}^{+}$cells and $\mathrm{Eya}^{-} \mathrm{Cas}^{+}$cells, which were also observed when expressing UAS-eya alone in mosaic clones. Thus the imperfect rescue may have been due to variations in the timing and level of Eya expression in these experiments.

Wnt affects Eya expression only transiently in region $2 \mathrm{~b}$, so some $d s h^{-}$polar and stalk cells form (Supplementary Fig. 9a, b). However, $0 / 75$ polar/stalk units analyzed were composed entirely of $d s h^{-}$cells compared to 6/82 in controls clones $(P=0.03$ by Fisher's exact test). When $d s h$ was reduced by $c 306-$ Gal4-driven
RNAi, which was expressed in a larger group of follicle precursor cells than in the mosaic clone experiments (Supplementary Fig. 9c), we observed $22-51 \%$ of ovarioles with at least one egg chamber fusion (Fig. 4j, Supplementary Fig. 9d), indicating a problem with producing the correct number of polar and stalk cells in the right location for egg chamber budding. UAS-eya overexpression led to an even stronger effect: $72-92 \%$ ovarioles with fused egg chambers as previously reported ${ }^{27}$.

Together these results show that the level of Wnt signalling that is normally present in region $2 b^{\mathrm{A}}$ of the germarium is a
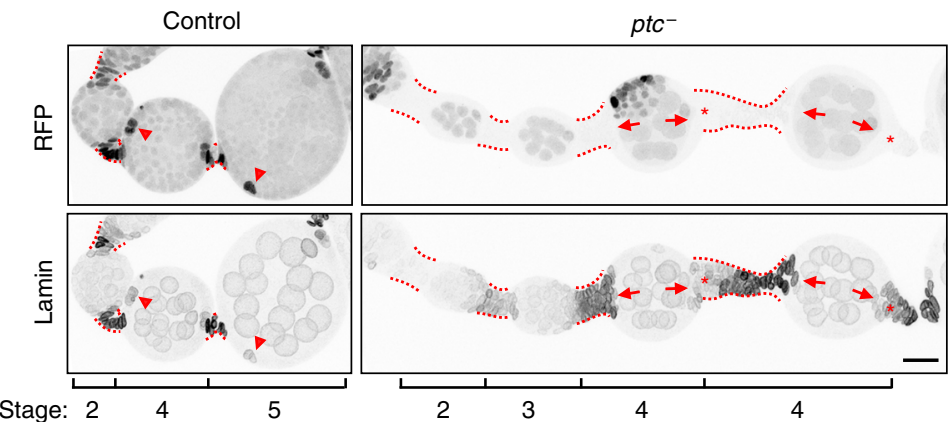

b

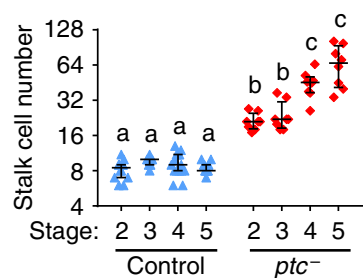

c
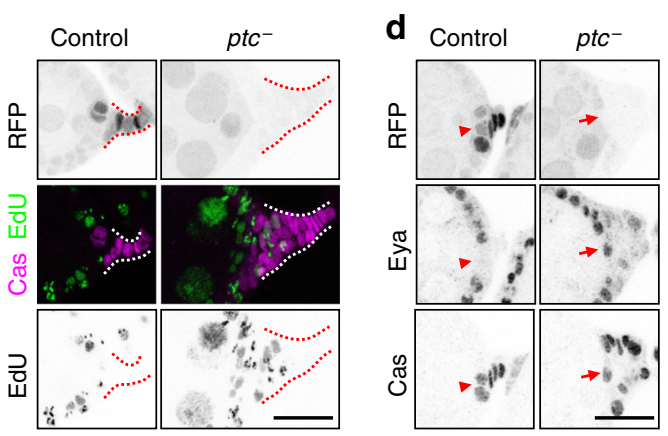

g
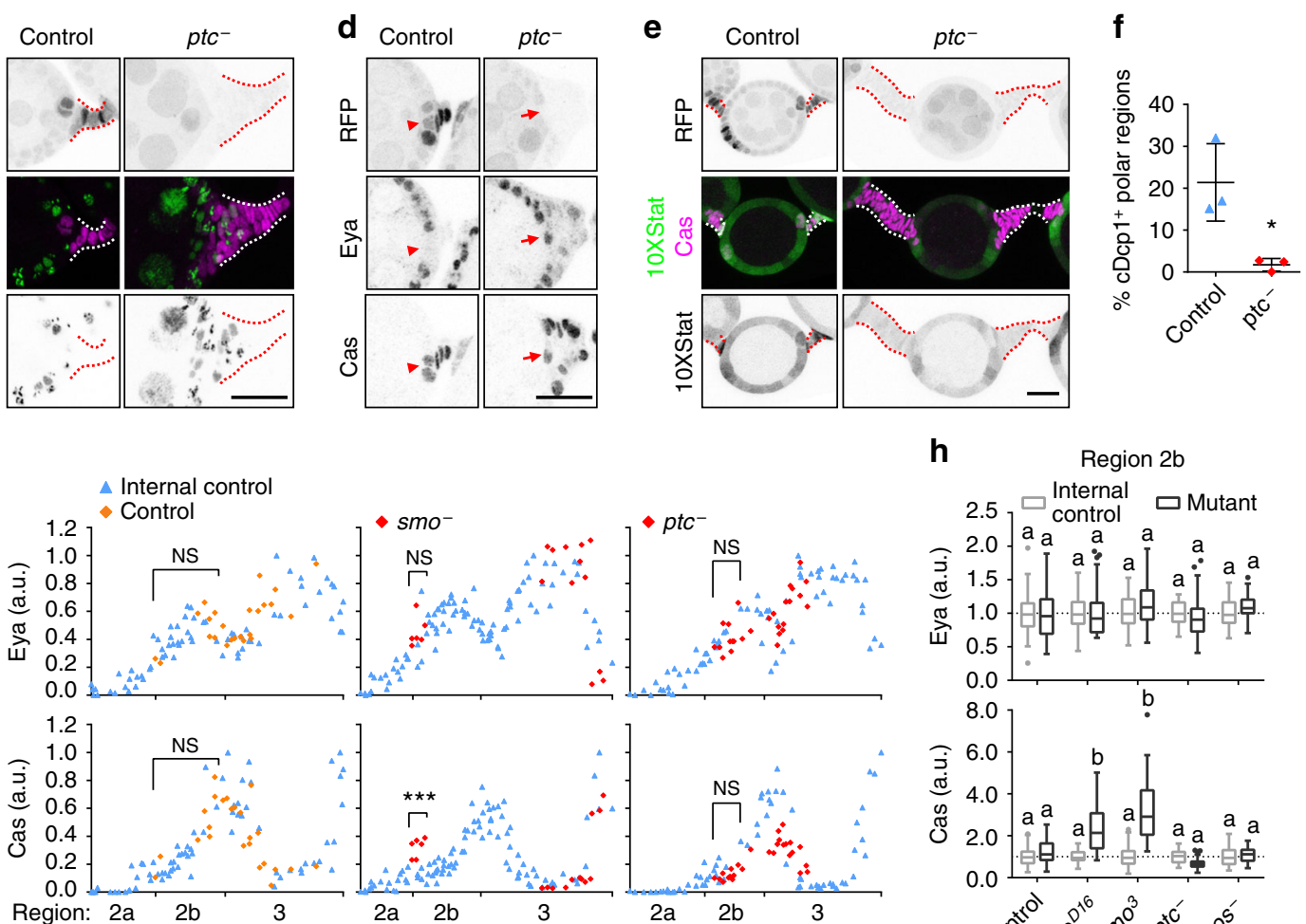

h

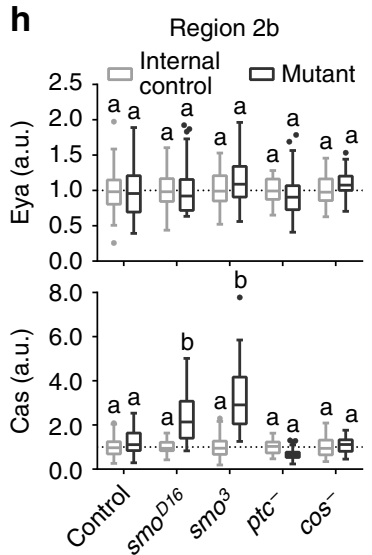

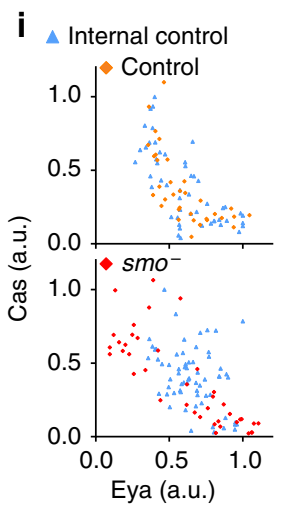

j

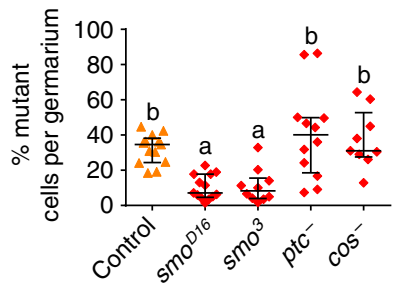

k

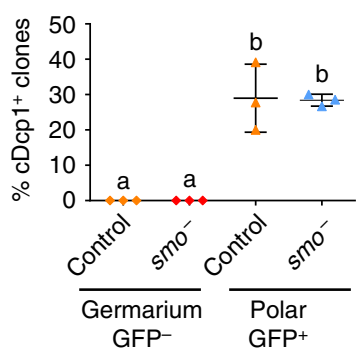

I

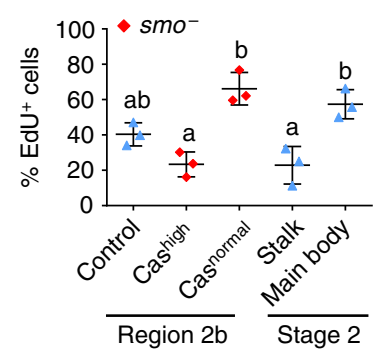


capable of repressing the main body cell fate determinant Eya. In $A x n^{-}$clones, persistent activation of Wnt signalling to the $2 \mathrm{~b}^{\mathrm{A}}$ level at later stages produces a strong bias towards polar and stalk cell fates. Nevertheless, polar and stalk cells can form in the absence of Wnt signalling, albeit less frequently than normal. Therefore, we conclude that Wnt signalling is a transient input into Eya expression and that there are normally multiple inputs into Eya expression and progenitor cell fates.

Hh signalling controls the timing of differentiation. If hyperactive Wnt signalling causes excess and ectopic polar and stalklike cells by inhibiting Eya and biasing cell fate, how does hyperactive Hh signalling cause both excess (Fig. 1e; Fig. 5a) and ectopic (Fig. 2e; Supplementary Fig. $10 \mathrm{a}^{32,36}$ ) polar and stalk-like cells while still producing main body cells (Figs 2e and 5a; Supplementary Fig. 10a)

We first analyzed the long stalk phenotype. The supernumerary stalk cell phenotype appeared most obvious when the majority of follicle cells associated with an egg chamber were mutant. In contrast to control stalks that contained a stable number of stalk cells ranging from 6 to 13, ptc stalks contained an average of 21 cells at stage 2 , and continued to increase in number over time (Fig. 5a, b). Another feature of stalk cell maturation is that they normally become physically separated from the polar cells (Fig. 5a, arrowhead), although stalk cells initially form at the poles. In contrast, $p t c^{-}$stalk cells remained associated with the poles, and with $\mathrm{Cas}^{+}$or Lamin $\mathrm{C}^{+}$(another stalk cell marker ${ }^{15}$ ) cells on the main body (Fig. 5a, arrow). Mature Cas high stalk cells are normally EdU-negative after stage 2 (Fig. 5c). In ovarioles with $p t c^{-}$clones in regions where stalk was still connected to polar and/or main body cells, many mutant cells were EdU positive (Fig. 5c), and Eya and Cas were sometimes coexpressed (Fig. 5d), suggesting that some of the $p t c^{-}$cells were multipotent precursors. Their low Lamin C expression level (e.g., asterisk in Fig. 5a) also resembled that of control precursors in the germarium. Therefore, excessive stalk cell production in $p t c^{-}$ clones seems due to persistence of precursor cells that continue contributing cells to the enlarging stalk.

We then asked how supernumerary polar cells form in response to Hh hyper-activation. Typically 3-6 polar cells form and all but two are eliminated by apoptosis ${ }^{35}$, a process that requires JAK/STAT signalling ${ }^{37}$. We observed a reduction of JAK/STAT activation in mutant polar cell regions (Fig. 5e), and reduced apoptosis of polar cells at stages 2-5 (Fig. 5f). Furthermore, some $p t c^{-}$cells in the main body region became Eya ${ }^{\text {low }}$ Cas ${ }^{\text {high }}$ (Supplementary Fig. 10a), and thus formed ectopic polar/stalk cells as they matured, also consistent with the idea of a differentiation delay.
If hyperactive Hh signalling causes delayed differentiation, loss of Hh signalling should expedite differentiation. Earlier studies have not detected a differentiation defect in $s m o^{-}$[21], but these studies did not have access to early cell fate markers and quantitative imaging. Using quantitative analysis of Eya and Cas in the germarium (Supplementary Movies 5-7), we observed that a subset of $s m o^{-}$cells showed significantly higher Cas expression in region $2 b$, or higher Eya expression in region 3 (Fig. $5 g-i$ ). The $s m o^{-}$clone size was significantly smaller than $p t c^{-}$or control in the germarium (Fig. 5j), which could be due to apoptosis, or to premature differentiation into polar/stalk-like cells, which stop dividing early as part of their normal development. Cells with reduced $\mathrm{Hh}$ signalling in the germarium rarely showed any cDcp1 signal, although polar cell regions were frequently positive for cDcp1 as expected (Fig. 5k), suggesting that apoptosis was not the explanation. Interestingly, EdU incorporation in the Cas high $s m o^{-}$cells was similar to the $\mathrm{Cas}^{+}$stalk cells in stage 2 , suggesting that a subset of $s m o^{-}$cells exited the cell cycle (Fig. 5l). Together, these results suggest that Hh signalling can suppress differentiation of follicle precursor cells.

We previously reported that some $s m o^{3}$ clones lack Cas expression and thus polar and stalk cells ${ }^{27}$. Consistent with this observation, we found that smo mutant polar and stalk cell clones occur less frequently than control polar and stalk cell clones and less frequently than smo mutant main body clones (Supplementary Fig. 10b, c). However, using multiple smo alleles, we also found that this effect is not fully penetrant, so some $s m o^{-}$clones express similar Eya and Cas as controls at stage 4 (Supplementary Fig. 10d, e). Since ptc mutant cells show delayed differentiation as opposed to the dramatic cell fate bias observed in Axn mutant cells, we conclude that the reduction in polar and stalk cells is likely an indirect effect secondary to defective follicle cell differentiation.

Wnt and Hh double mutants show additive effects. If Wnt and Hh have distinct and independent effects on follicle precursor cell differentiation, hyper-activation or loss of both Wnt and $\mathrm{Hh}$ should show additive effects. Indeed, whereas a normal stalk contains 8-10 cells, we observed an average of 30 stalk cells in Axn or cos single mutants, and $\sim 60$ stalk cells in $A x n$ and cos double knockdown cells (Fig. 6a, b). The $A x n$ and cos double mutant cells are also largely biased towards $\mathrm{Cas}^{+}$polar/stalk-like cells (Fig. 6a), suggesting that the $\mathrm{Eya}^{+} \mathrm{Cas}^{+}$precursors that accumulated due to delayed differentiation preferentially adopted a polar/stalk fate in the presence of high Wnt signalling. Loss of both Wnt and Hh also showed a more extreme phenotype than either alone. Combined loss of Wnt and Hh activity using c306Gal4 to drive smo and dsh RNAi in the follicle precursor cells increased the frequency of egg chamber fusions to $80 \%$ compared

Fig. 5 Hh signalling controls the timing of differentiation. a 3D projection view of ovarioles with ptc ${ }^{S 2}$ heterozygous control or large homozygous mutant $\left(\mathrm{RFP}^{-}\right)$FSC clones. Dashed lines mark the stalk region. Arrowheads point to control polar regions, arrows point to mutant regions. Asterisks marks mutant stalk cells proximal to posterior polar cells where Lamin is low. b Anterior stalk cell number in ptc $\mathrm{S}^{2}$ heterozygous control or large homozygous mutant FSC clones. Data (median with interquartile range) from 8-15 egg chambers. $P<0.0001$. c, d Sagittal view of posterior polar/stalk region in ptc 52 heterozygous control or homozygous mutant egg chambers. e STAT activity shown by $10 X S t a t-G F P$ in ptc ${ }^{S 2}$ heterozygous control or homozygous mutant stalk cell regions. f Clones with $\mathrm{cDcp} 1$ in $\mathrm{ptc}^{\mathrm{S2}}$ heterozygous control or homozygous mutant polar cell regions. Data (mean \pm s.d.) from 3 experiments, $194-466$ stage 2-5 polar cell regions. ${ }^{\star} P<0.05$ (Unpaired $t$-test). $\mathbf{g}$ Eya and Cas intensity in germaria with FRT40A control, smo ${ }^{3}$, or ptc ${ }^{S 2}$ mutant clones compared to control cells in the same germarium. ${ }^{\star \star \star} P<0.001$ (Unpaired $t$-test). $\mathbf{h}$ Eya and Cas intensity in germarium region $2 \mathrm{~b}$. Data (median with interquartile range) from 21 to 68 cells from 4-6 germaria per genotype. $P<0.001$. i Eya and Cas intensity containing FRT40A control or smo ${ }^{3}$ mosaic FSC clones in germarium region 3. Data from 85-105 cells from 3 germaria per group. j Mutant clone size. Data (median with interquartile range) from $9-13$ germaria, $P$ $<0.01$. k Clones with cDcp1 in FRT40A control or $\mathrm{smo}^{3}$ homozygous mutant clone (GFP ${ }^{-}$) in the germaria and $\mathrm{GFP}^{+}$control cells in stage 1-2 polar/stalk regions. Data (mean \pm s.d.) from 3 experiments, 39-51 ovarioles per genotype, $P<0.001$. I EdU ${ }^{+}$cells in smo ${ }^{3}$ heterozygous control or homozygous mutant. Data (mean \pm s.d.) from 3 experiments, 1222, 112, 119, 196, and 719 cells, $P<0.01$. Scale bars, $20 \mu$ m. Samples labelled with different letters are significantly different using ANOVA with Turkey's test $\mathbf{b}, \mathbf{k}$, I, or Kruskal-Wallis with Dunn's test $\mathbf{h}$, $\mathbf{j}$ 
a

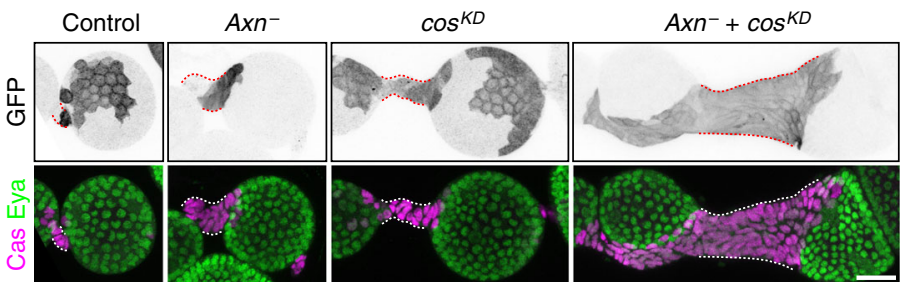

C

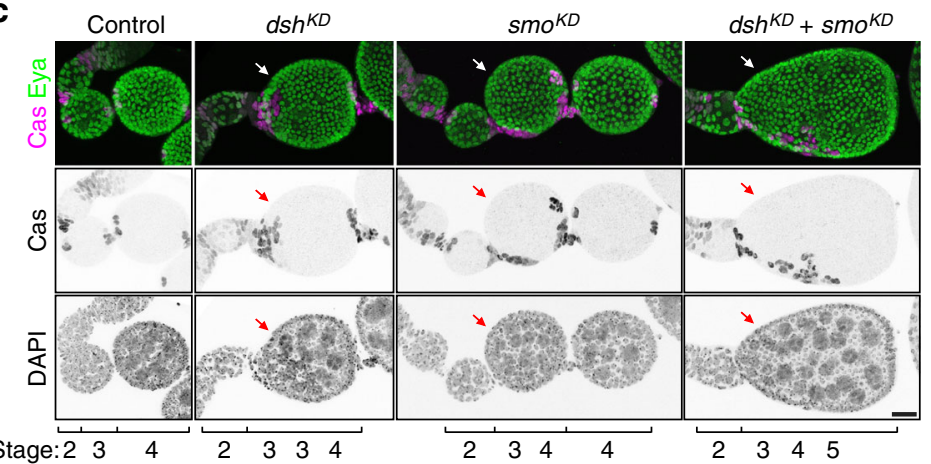

e
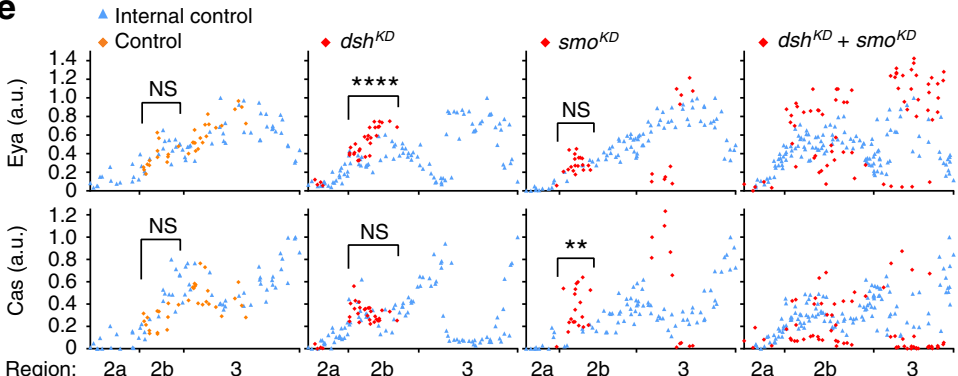

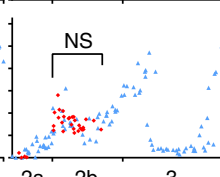

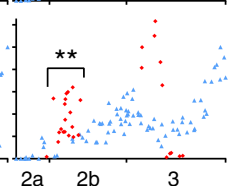

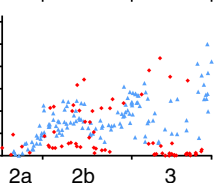

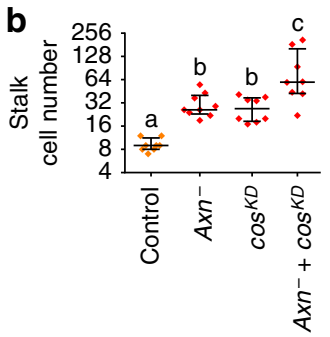

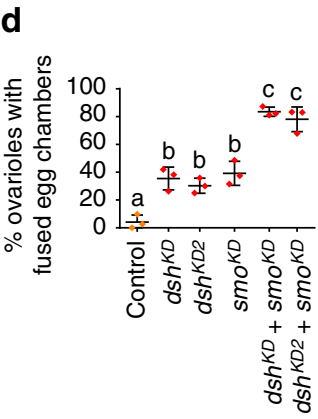

f

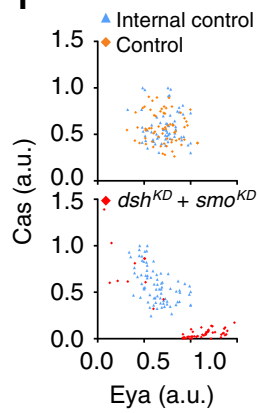

Fig. $6 \mathrm{Wnt}$ and Hh double mutants show additive effects on egg chamber patterning. a 3D projection view of one half of stage 4 egg chambers with FRT82B control, $A x n^{5044230}$, cosRNAi, or $A x n^{5044230}+\cos R N A i$ mosaic FSC clones. Dashed lines mark the stalk region. $\mathbf{b}$ Quantification of stalk cells anterior to stage 4-5 egg chambers in FRT82B control, $A x n^{5044230}$, cosRNAi, or Axn ${ }^{5044230}+$ cosRNAi mosaic FSC clones. Data (median with interquartile range) from $n=8-9$ egg chambers per genotype. $P<0.01$. c 3D projection view of ovarioles with stage 2-5 egg chambers in c306-Gal4 control or dshRNAi and/or smoRNAi. Arrows point to fused egg chambers. d Quantification of fused egg chamber rate in c306-Gal4-driven knockdown of dsh and/or smo in follicle precursor cells. Data (mean \pm s.d.) from $n=3$ experiments, $145-189$ ovarioles per genotype. $P<0.01$. e Eya and Cas intensity in germaria with AyGal4 control (orange diamonds), dshRNAi and/or smoRNAi mutant clones (red diamonds) compared to control cells in the same germarium (blue triangles). ${ }^{\star \star} P$ $<0.01$; ${ }^{* \star \star \star} P<0.0001$ (Unpaid $t$-test). f Eya and Cas intensity in germarium region $2 b^{P}$ containing AyGal4 control or dshRNAi+smoRNAi mutant FSC clones. Data from 130-134 cells from 3 germaria per group. Scale bars, $20 \mu \mathrm{m}$. Samples labelled with different letters are significantly different using ANOVA with Turkey's test $\mathbf{b}, \mathbf{d}$

to $30-40 \%$ for the single knockdowns (Fig. $6 \mathrm{c}, \mathrm{d}$ ). In the germarium, double mutant cells showed signs of both increased Eya and premature differentiation into Eyahigh $\mathrm{Cas}^{\text {low }}$ and Eya ${ }^{\text {low }}$ Cas high cells (Fig. 6e, f; Supplementary Movies 8-11).

Notch signalling promotes differentiation in multiple regions. The involvement of Wnt and $\mathrm{Hh}$ in follicle precursor cell differentiation prompted us to ask how they relate to Notch signalling, a pathway known to be involved in ovary development at multiple stages $8,9,38$. We used a Notch activity reporter, the Notch responsive element (NRE) driving expression of $\mathrm{GFP}^{39}$ to address this question. NRE-GFP showed a basal level in all cells, but peaked in a subset of cells, in the region $2 \mathrm{a} / 2 \mathrm{~b}$ boundary cells and in polar cells (Fig. 7a, b). This is consistent with the known roles of Notch in the formation of the germline stem cell niche ${ }^{40,41}$, cross-migration of FSC daughters ${ }^{8}$, and polar cell specification $^{9}, 42$. Although the $2 \mathrm{a} / 2 \mathrm{~b}$ boundary signal was observed in only a subset of ovarioles, we confirmed that it depended on Notch, using NotchRNAi flip-out clones (Fig. 7c, d,
Supplementary Fig. 11). Next, we asked whether Notch activity changed upon loss or hyper-activation of Wnt or Hh. We did not observe any change in Notch activity in germarium region $2 \mathrm{~b}$ $d s h^{-}, A x n^{-}$, or $s m o^{-}$clones although it was reduced in $p t c^{-}$cells (Supplementary Fig. 12a, b). $A x n^{-}$clones showed Notch activity comparable to control polar cells both at egg chamber termini and at ectopic locations that were directly contacting the germline, suggesting that high Wnt signalling predisposes cells to specification as polar cells by Notch (Supplementary Fig. 12c). Polar cells show patchy NRE expression as reported previously ${ }^{43}$. We found the same percentage of $A x n^{-}$cells showing NRE (66\%, $n=91 A x n^{-}$polar cells) compared to control polar cells $(65 \%$, $n=177$ control polar cells). Consistent with the conclusion that Hh signalling prevents premature differentiation of follicle cells in the germarium, $s m o^{-}$cells show precocious Notch activity in region 2b/3 (Supplementary Fig. 12a, d), while ptc ${ }^{-}$cells show delayed Notch activity in the polar cell regions (Supplementary Fig. 12e).

To assess the effect of Notch signalling on follicle precursor development, we quantified the Eya and Cas levels in Notch ${ }^{-}$and 
NotchRNAi FSC clones (Supplementary Movies 12-13). Although we previously did not detect an obvious change in Eya or Cas in the germarium ${ }^{27}$, with our new quantitative measurements we detected a slight decrease in Cas and a minor increase in Eya in region $2 \mathrm{~b}$ (Fig. $7 \mathrm{e}, \mathrm{f})$. The Notch receptor modifier fringe (fng) mutant ${ }^{44}$ showed a similar phenotype as loss of Notch (Fig. 7f). In region 3, Notch ${ }^{-}$cells showed higher Eya and lower Cas in the polar/stalk region (Supplementary Fig. 13a). The change of Eya and Cas is consistent with the role of Notch in promoting both precursor cross-migration and differentiation, as well as polar cell specification, and we observed a high frequency of fused egg chambers when NotchRNAi clones covered the anterior polar cell region (Supplementary Fig. 13b).

Although loss of Notch activity causes loss of polar cells and egg chamber fusion ${ }^{45}$, hyper-activation of Notch by expressing the UAS-N.intra did not alter Eya or Cas levels in region $2 \mathrm{~b}$ (Fig. 7e, f; Supplementary Movie 14), or induce ectopic polar/stalk cells on the main body region in stage 4 egg chambers

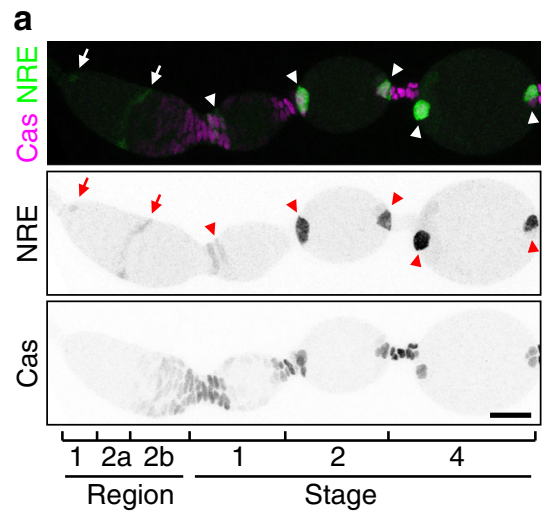

e

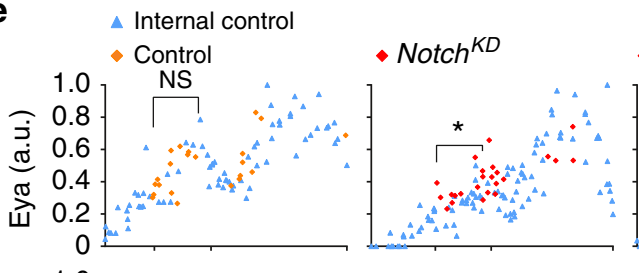

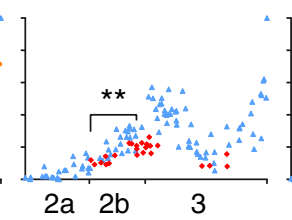
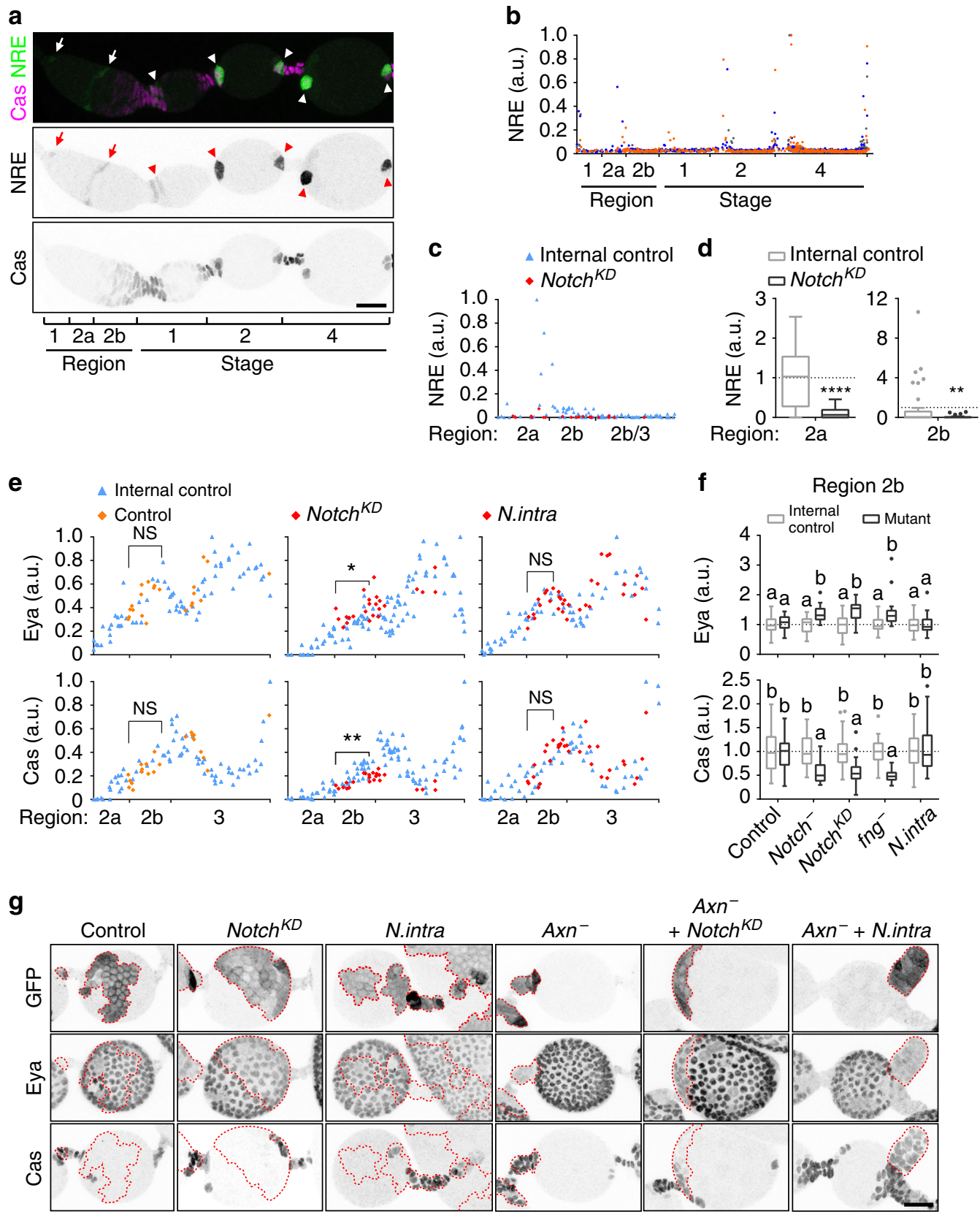

h

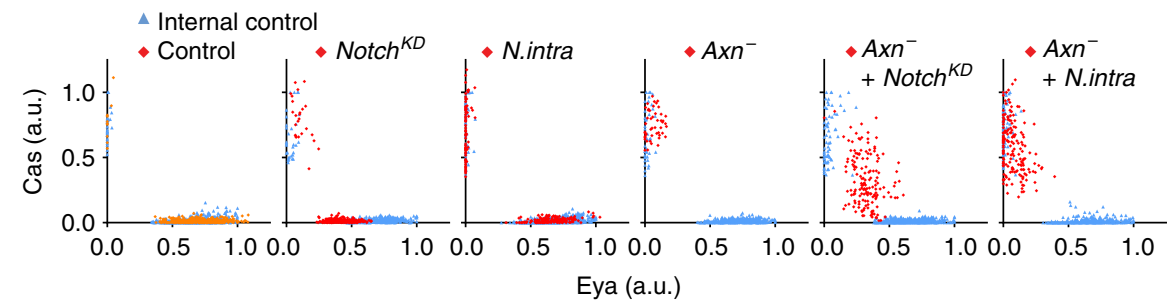


(Fig. $7 \mathrm{~g}^{10,12}$ ), suggesting that Notch activity is not sufficient for polar cell specification. Hyper-activation of Wnt together with hyper-activation of Notch caused an even larger number of polar and stalk-like cells (12.5\%), compared to hyper-activation of Wnt (3.6\%) or Notch (5.5\%) alone (Fig. $7 \mathrm{~h})$. We noticed that polar cells normally express less Cas than stalk cells $(82 \pm 13 \%$ vs. $100 \pm 13 \%$ comparing 17 polar and 25 stalk cells in control stage 4 egg chambers, $P<0.001$ ), and constitutive Notch activity in $A x n^{-}$increased the number of cells expressing polar as well as stalk cell Cas levels (Fig. 7h). Hyper-activation of Wnt together with loss of Notch caused follicle cells to show Eya and Cas patterns typical of precursor cells in stage 1 (Figs $2 \mathrm{~b}$ and $7 \mathrm{~h}$ ), suggesting that Notch is necessary for differentiation of polar and stalk-like cells specified by Wnt signalling.

Interestingly, Notch appeared to promote further differentiation in multiple cell types even in stage 2-8 egg chambers. For example, NotchRNAi in the posterior pole cell caused Cas ${ }^{+}$cells to abnormally express Eya (Supplementary Fig. 13c, d). Conversely loss of Notch in main body cells reduced the Eya level in stage 4 when Eya is normally increasing. In both cases, the observation is consistent with defective differentiation[38]. Furthermore, later in development the Eya level normally decreases again in main body cells and NotchRNAi prevented this (Supplementary Fig. 13e). NotchRNAi expressing stalk cells did not show the normal intercalated morphology (Supplementary Fig. 13c, d). Together, these results suggest that Notch promotes differentiation in multiple stages and cell types.

\section{Discussion}

When adult tissue stem cells divide asymmetrically to self-renew and produce a daughter cell that commonly becomes a transitamplifying precursor, the stem cell might retain its character by virtue of its proximity to niche signals whereas the transient amplifying precursors might acquire their properties due to displacement from the niche. Alternatively, niche signals might continue to influence precursor cell fate and differentiation. Here we report a previously undescribed Axn mutant phenotype that reveals a potent effect of Wnt signalling on follicle cell fates and therefore implicates Wnt signalling in diversification of follicle cell precursor fates as they leave the niche. We found graded responses to both Wnt and $\mathrm{Hh}$ and distinct actions of these niche signals, which together influence the development of the transitamplifying follicle precursor population. Combining earlier studies on Wnt and Hh in follicle stem cell maintenance ${ }^{15,16,46}$ with this study, Wnt and Hh resemble morphogens that specify FSC fate at high concentration, a multipotent precursor fate at a lower concentration, main body precursor fate in the region lacking Wnt and low in Hh signalling, and differentiated cell fates in the absence of both Wnt and Hh. This proposal is consistent with a recently published study proposing that escort cells, which reside in the domain of highest Wnt activity, depend on Wnt for their fate ${ }^{47}$.

Earlier studies reported egg chamber formation defects due to reducing the Wnt or Hh ligand levels ${ }^{13}, 15,32$. However, recent studies show that Wnt and $\mathrm{Hh}$ in escort cells also affect germline differentiation ${ }^{19}, 20,48-51$; therefore any defects from reduction of Wnt or Hh ligands could either be due to effects on the germline and/or follicle cells. We clarified this issue by reducing Wnt and Hh intracellular signalling components directly in follicle cells in mosaic clones, or by RNAi knockdown specifically in follicle precursor cells.

In the germarium, the follicle precursor cells in regions $2 \mathrm{~b}$ and 3 contain both specified and unspecified cells ${ }^{8}$, yet previous studies lacked cell fate markers and quantitative methods to assess the influence of different signalling inputs. Our quantitative analyses of Eya and Cas distinguish cell states in region $2 \mathrm{~b}$, where both markers are low but increasing, which we propose identifies a precursor state. In region 3/stage 1 , fates begin to diverge into Eya ${ }^{\text {low }}$ Cas high cells capable of producing polar or stalk cells and Eyahigh $\mathrm{Cas}^{\text {low }}$ cells likely committed to main body fate. This combination of markers not only provides a sensitive and detailed description of the FSC differentiation process, but also reveals early changes in the germarium that were not previously detected. First, alterations in Wnt activity affect the expression of Eya, a key main body fate determinant ${ }^{23}$. Second, reduction of $\mathrm{Hh}$ activity in $s m o^{-}$cells resulted in increased levels of Cas in region $2 \mathrm{~b}$, which we suggest reflects premature differentiation. Although the Eya level did not increase in region $2 \mathrm{~b} \mathrm{smo}^{-}$cells, this is likely because of inhibition of Eya by Wnt in this location. Third, reduction of Notch activity caused cells in region $2 \mathrm{~b}$ to express slightly more Eya and less Cas than control cells, providing a possible explanation for how Notch promotes polar cell fate.

We show that hyper-activation of Wnt signalling biases follicle cells to polar and stalk cell fates, while loss of Wnt signalling causes a weak bias against polar and stalk fates. Why does loss of negative regulators produce a stronger effect than loss of positive ones? One theoretical possibility is that loss of negative regulators produces an extremely high and non-physiological level of signalling. However, our reporter data show that Wnt activity in $A x n^{-}$cells is within the normal range found in region $2 \mathrm{~b}$ (Fig. 2c). A second possibility is that the levels and distribution of negative regulators might be important factors shaping the Wnt response in that region. Unfortunately, this cannot be tested because available antibodies against Axn are not sensitive enough to detect the endogenous protein. A third possibility, which is not mutually exclusive, is that other factors ${ }^{52}$ work together with Wnt to inhibit Eya expression and specify polar and stalk cell fates in normal development.

Fig. 7 Notch is permissive but not instructive for differentiation of multiple cell types. a NRE-GFP pattern from the germarium to stage 4 egg chambers. Arrows point to Notch activity in the cap cells and region $2 \mathrm{a} / 2 \mathrm{~b}$ boundary cells and arrowheads point to polar cells. b Quantification of NRE-GFP intensity in cap cells, escort cells, and follicle cells along the anterior-posterior axis until stage 4. Data from $n=1533$ cells in 3 ovarioles. Different colours represent different ovarioles. Data were normalized to maximum NRE-GFP intensity per sample. c NRE-GFP in germaria with NotchRNAi mutant clones (red diamonds) compared to control cells in the same germarium (blue triangles). d Quantification of NRE-GFP in germaria region $2 a$ and $2 b$ with mosaic clones. Data (median with interquartile range) from $n=20-66$ cells from 3 germaria. Data were normalized to average NRE intensity in internal control cells. ${ }^{\star \star} P<0.01,{ }^{\star \star \star \star} P<0.0001$ (Mann-Whitney test) e Eya and Cas intensity in germaria with FRT19A control (orange diamonds), NotchRNAi, or UAS-N. intra mutant clones (red diamonds) compared to control cells in the same germarium (blue triangles). ${ }^{\star} P<0.05$, ${ }^{\star \star} P<0.01$ (Unpaired $t$-test), $\mathbf{f}$ Quantification of Eya and Cas intensity in germarium region 2 b containing FRT19A control, Notch ${ }^{55 e 11}$, NotchRNAi, fng ${ }^{13}$, or UAS-N.intra mutant FSC clones. Data (median with interquartile range) from $n=17-60$ cells from 4 germaria per genotype. Data were normalized to average Eya or Cas in internal control cells. Samples labelled with different letters are significantly different at $P<0.01$ (Kruskal-Wallis with Dunn's test). $\mathbf{g}$ 3D projection view of one half of

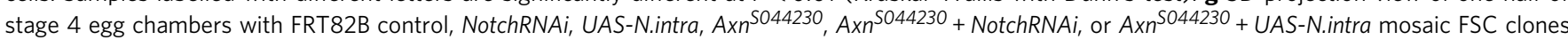
(GFP ${ }^{+}$, dashed lines). Eya ${ }^{+} \mathrm{GFP}^{-}$cells appear in the outlined $A x n^{5044230}$ clone due to $Z$ stack projection. $\mathbf{h}$ Quantification of Eya and Cas intensity in follicle cells with FRT82B control, NotchRNAi, UAS-N.intra, Axn ${ }^{5044230}$, Axn $n^{5044230}+$ NotchRNAi, or Axn ${ }^{S 044230}+$ UAS-N.intra mosaic FSC clones. Data from $n=$ 1051-1420 cells from 4 stage 4 egg chambers per genotype. Scale bars, $20 \mu \mathrm{m}$ 
Our results together with earlier reports ${ }^{21}$ support the idea that the graded response to $\mathrm{Hh}$ in the germarium serves to postpone differentiation. In $p t c^{-}$cells differentiation is postponed so long that when it finally does occur, it is in absence of Wnt. Most cells therefore acquire a main body fate but a few cells can seemingly randomly acquire a Cas high polar or stalk-like cell fate, as observed in stage 4-7 ptc or $\cos$ mutant clones (e.g., Fig. 2e, g; Supplementary Fig. 7a). This propensity may also explain the observation that some $d s h^{-}$cells adopt polar and stalk fates. In contrast, in the presence of high Wnt signalling, as in Axn and cos double mutant cells, most of the cells adopt polar/stalk-like fates (Fig. 6a, b).

We suggest two explanations for how Notch might promote polar cell fate in the germarium. First, Notch has been shown to promote cross-migration of one FSC daughter ${ }^{8}$. Our results suggest that this will cause the precursor to remain exposed to a high level of Wnt for longer and therefore to remain low in Eya compared to a posteriorly displaced precursor cell. Second, we suggest that Notch promotes differentiation, which leads to the observed increase in Cas expression in region 2b. Although essential for polar cell specification, hyper-activation of Notch is not sufficient since constitutive activation of Notch causes excess polar cells to form only at the two poles rather than on the main body region ${ }^{10,12}$. This implies that additional information is required besides Notch activity for polar cell fate. Here we report that short-range Wnt signalling maintains the potential for polar and stalk fates. Notch activity then acts on Eya ${ }^{\text {low }}$ precursor cells to specify polar cells and polar cells in turn express Upd to induce stalk cell fate ${ }^{9}$ in competent (i.e., Eya ${ }^{\text {low }}$ ), neighbouring cells.

Open questions remain. It is not entirely clear at what stage(s) polar cell fates become specified ${ }^{8,9}$. Since the clones we analyzed were made in FSCs, it is possible that fluctuations in Wnt signalling within the FSC as it produces precursor cells could influence their fates-alternatively or in addition to the spatially graded response that we measured. The presence of ectopic Eya ${ }^{-}$ $\mathrm{Cas}^{+}$cells in the fused egg chambers caused by the combined knockdown of $d s h$ and smo shows that the polar/stalk fate can develop in the absence of Wnt and Hh, suggesting either that the knockdown is incomplete, that other factors are present that can promote $\mathrm{Eya}^{-} \mathrm{Cas}^{+}$fates, or that in the absence of normal signals follicle cell fates are unstable and can randomly tip towards main body or polar/stalk. The high frequency of fused egg chambers in the double knockdowns does confirm the importance of these pathways for the normal spatial and temporal patterning of cell fates.

Wnt, Hh and Notch are common players in many adult stem cell systems including the skin, gut, and blood ${ }^{53-55}$, which all possess a transit-amplifying progenitor pool close to the stem cell niche. Our finding of the separable functions of Wnt, $\mathrm{Hh}$, and Notch in precursor cell fate specification, differentiation, and apoptosis provides an integrated model for how multiple signalling inputs produce the appropriate numbers and types of differentiated cells. The additive effects of hyper-activation of multiple signalling pathways described here may have implications for other adult stem cells, including cancer stem cells ${ }^{56}$.

\footnotetext{
Methods

Drosophila genetics and mosaic clone induction. Fly strains used in this study are listed in Supplementary Table 1. Fly genotypes used in each experiment are listed in Supplementary Data 1 . Stocks were maintained at room temperature. Crosses were initiated at room temperature and transferred to $25^{\circ} \mathrm{C}$ at $2-3$ instar larvae stage. For c306-Gal4; tubGal80ts experiments, adult female flies were transferred to $29^{\circ} \mathrm{C}$ for 7-10 days after eclosion. Egg chamber stage was determined based on germ cell nucleus diameter listed in Supplementary Table 2.

Mosaic clones were generated using the FLP/FRT system. 8-9 newly eclosed adult female flies (1-2 days old) along with 8 males were collected in a vial with wet yeast paste (dry yeast and water $1: 1.5$ ) and dry yeast and kept at $25^{\circ} \mathrm{C}$. Flies were
}

flipped without $\mathrm{CO}_{2}$ to a fresh vial daily until dissection, and heat shocked 2 days after collection. Males were added if $<3$ were present to ensure optimal ovary development. For making FSC clones up to stage 5, flies were heat shocked twice for $1 \mathrm{~h}$, about $4 \mathrm{~h}$ apart, in a $37^{\circ} \mathrm{C}$ water bath, and then were kept at $25^{\circ} \mathrm{C}$ for 5-7 days before dissection. For RNAi knockdown experiments, flies were transferred to $29^{\circ} \mathrm{C}$ after heat shock (except for eyaRNAi and armRNAi, which were kept at $25^{\circ} \mathrm{C}$ ). For making FSC clones up to stage 8 , flies were kept for 6-8 days before dissection. For border cell clones in Fig. 1d, flies were heat shocked once for $30 \mathrm{~min}$ and kept for 4-5 days before dissection. For negative mosaic clones, we excluded the false clones due to damage that contain condensed Hoechst staining and diffused nuclei signals. Polar and stalk cells always show higher ubiRFP/GFP signals and therefore the intensity is not an indication of twin spot in those regions. For MARCM clones, we observed some leaky GFP expression in follicle cells in stage 6 and later, likely due to actinGal 4 being too strong in stage 6 and later such that tubGal 80 was not able to suppress all Gal4 activities. Therefore, we only analyzed MARCM clones before stage 6 .

Immunostaining and EdU incorporation. Adult female ovaries were dissected in Schneider's Drosophila medium (Thermo Fisher Scientific, Waltham, MA) with $20 \%$ fetal bovine serum and transferred to a $0.6 \mathrm{ml}$ microfuge tube with $100 \mu \mathrm{l}$ dissection medium. Ovaries were dissociated by pipetting up and down $\sim 50$ times using a $200 \mu \mathrm{l}$ pipette set to $50 \mu \mathrm{l}$. Dissociation in this way causes random physical damage to the egg chambers ${ }^{57}$, but we found it more efficient than pulling ovarioles out of the muscle sheath using forceps, which causes more damage to the germarium or younger egg chambers. Ovarioles were immediately fixed for $20 \mathrm{~min}$ in $4 \%$ paraformaldehyde at $4{ }^{\circ} \mathrm{C}$. After fixation, ovarioles were washed with $\mathrm{PBS} / 0.4 \%$ Triton X-100 (PBST), and then incubated with primary antibodies overnight at $4{ }^{\circ} \mathrm{C}$. The following day, ovarioles were washed with PBST before incubation in secondary antibody for $1.5-2 \mathrm{~h}$. After removal of secondary antibodies, samples were stained with Hoechst for 20 min. Samples were washed in PBST before sorting in PBST. Sorting was conducted by using forceps under a dissection microscope to remove mature eggs and clustered ovarioles from a given sample for optimal mounting. Without sorting, mature eggs make it difficult to compress the sample, the germaria can be tilted, and clustered ovarioles often overlap each other rendering imaging difficult. After sorting, samples were stored in VECTASHIELD (Vector Laboratories, Burlingame, CA) at $4^{\circ} \mathrm{C}$.

The following antibodies were used in this study: chicken anti-GFP (1:2000, Abcam, Cambridge, UK; 13970) (used to amplify MARCM GFP, flip-out GFP, Ptcpelican-GFP, and NRE-GFP, not used on negative mosaic ubi-GFPnls), rabbit antidsRed (1:1000, Takara Bio USA, Mountain View, CA; 632496) (used to amplify flip-out RFP and NRE-RFP, not used on negative mosaic ubi-RFPnls or Fz3-RFP), mouse anti-Eyes Absent (1:50-200, Developmental Studies Hybridoma Bank (DSHB), Iowa City, IA; 10H6, needs pre-absorption if staining is noisy), mouse anti-Fascillin III (1:50, DSHB 7G10), rat anti-E-cadherin (1:50, DSHB DCAD2), rabbit anti-Castor (1:5000, Ward F. Odenwald $\left.{ }^{58}\right)$, mouse anti-Armadillo (1:100, DSHB N27A1), mouse anti-Smoothened (1:4, DSHB 20C6), rat anti-Cubitus interruptus (1:10, DSHB 2A1), rabbit anti-cleaved Drosophila caspase 1 (1:200, Cell Signaling Technology, Danvers, MA; 9578), mouse anti-Notch intracellular domain (1:200, DSHB C17.9C6), and mouse anti-Lamin C (1:200, DSHB LC28.26).

For EdU incorporation, adult female ovaries were dissected in Schneider's Drosophila medium with $20 \%$ fetal bovine serum and transferred to a microfuge tube with the dissection medium plus $40 \mu \mathrm{M}$ EdU, and kept at room temperature on a shaker for $1 \mathrm{~h}$. Ovarioles were then dissociated, fixed, and stained with primary and secondary antibodies as described above. Before staining with Hoechst, an EdU detection reaction was performed according to the manufacturer's manual (Thermo Fisher Scientific).

Imaging and image processing. Due to the spherical organization of the egg chambers, few follicle cells have their nuclei located on the same imaging focal plane. Therefore, we imaged the egg chambers in full $Z$ stacks. Samples were mounted on a glass slide in VECTASHIELD $(25 \mu \mathrm{l}$ for early stage ovarioles, or $65 \mu \mathrm{l}$ for stage 9/10) using a $22 \mathrm{~mm} \times 40 \mathrm{~mm}$ cover glass, to ensure that the germarium was mounted flat, but not compressed, and that later stages were compressed to a consistent degree. All images were taken on a Zeiss LSM780 confocal microscope, using a $40 \times 1.4$ N.A. oil objective. $Z$ stacks covering the entire germaria or ovarioles were taken with a $0.43 \mu \mathrm{m}$ step size for germarium and ovarioles, or a $1 \mu \mathrm{m}$ step size for border cell clusters. XY resolution is $0.14 \mu \mathrm{m}$ for germaria, or $0.35 \mu \mathrm{m}$ for ovarioles. Laser power corrections were applied by increasing the laser power as the objective scans from the top of the sample to the bottom of the sample, so that the signal on the bottom did not appear weaker than the top.

3D images were visualized in Imaris (Bitplane, South Windsor, CT), and annotated in Excel (Microsoft, Redmond, WA), to categorize the developmental stage, sample condition, mounting condition, imaging condition, clone location, and result interpretation. Developmental stage was determined as described above. Sample condition includes whether they were damaged, or still tightly packed in muscle sheath. Severely damaged egg chambers had an incomplete follicle epithelium and leaky germ cells, or large patches of follicle cells without nuclear stain. Mild damage caused a small patch of follicle cells to show condensed Hoechst staining, and diffused or reduced nuclei Eya, Cas, or ubi-GFP/RFPnls signal ${ }^{57}$. Samples with severe damage were not analyzed, and the damaged cells in a sample 
with mild damage were not included in the analysis. We preferred to analyze samples out of the muscle sheath, because their morphology was not affected by squeezing from neighbouring egg chambers. Samples tightly packed in muscle sheath were not used for intensity measurement because it was difficult to perform laser power correction. Mounting condition denotes if the sample was too compressed or too tilted. If the germarium was too compressed, the germline cysts were squeezed and it was difficult to perform $3 \mathrm{D}$ rotation as described below. If too tilted, laser correction became difficult. Imaging condition marks whether the image was taken with proper laser power correction. This was estimated by comparing the signal intensity of the top, middle, and bottom of the sample visually, and was quantified as described below. Clone location and result interpretation were listed to help summarize the results, draw conclusions based on the phenotype seen across multiple ovarioles, and select representative images for presentation.

Representative images were exported from Imaris using either Easy 3D view or slice view. Since different follicle cell nuclei were located on different focal planes, 2-5 $\mu \mathrm{m} Z$ stacks were used to show single follicle cell layers, while $12-25 \mu \mathrm{m} Z$ stacks were used to show one half of the egg chambers. Exported images were rotated and cropped in Photoshop (Adobe, San Jose, CA). Single channel images were converted from a black background to a white background using Invert LUT function in Fiji ${ }^{59}$.

3D quantification. Image segmentation was performed using Imaris. First, samples were rotated using the Free Rotate function. Egg chambers were rotated so the polar cells aligned horizontally, with the anterior to the left. Germaria were rotated in two steps. The first step positioned region $2 \mathrm{~b}$ cysts vertically in the Z-direction by placing an Oblique Slicer in the mid-sagittal section of the germaria, and performing free rotation to the orthogonal view of the oblique slicer. The second step rotated the germaria anterior to the left to place region $2 \mathrm{~b}$ cysts vertically in XYdirection. Second, follicle cell nuclei were detected using the Spots function. For the germaria, a $2.5 \mu \mathrm{m}$ diameter spot size was used for automatic spot detection in the channel with follicle cell nuclei signals. Spots were then manually edited so that each follicle cell was marked. Dividing, dying, or damaged cells showed clear signs, including condensed Hoechst staining and diffused, or reduced, nuclei Eya, Cas, or ubi-GFP/RFP signal, and were not quantified. The $2.5 \mu \mathrm{m}$ spots were then used to create a masked channel, and automatic spots detection based on that channel was applied to create $1.75 \mu \mathrm{m}$ spots, so that only the centre of the nuclei with a strong and even signal was used for quantification. For egg chambers, a $3.46 \mu \mathrm{m}$ diameter spot size was used for automatic spot detection, followed by reduction to $1.75-2$ $\mu \mathrm{m}$. Third, background intensities were estimated by placing 8-12 1.75-2 $\mu \mathrm{m}$ spots in two $Z$ planes in same region as the measured follicle cells. For Eya and Cas, background spots were placed in the germ cell cytoplasm, while for Wnt or Hh reporters they were placed in the region $2 \mathrm{~b}$ germ cell nuclei. Fourth, accuracy of laser power correction was determined by selecting control cells at the top, middle, and bottom of the germarium or egg chamber in the same region, and comparing their signal intensities.

Data for spot position and channel mean intensity were exported from Imaris, and processed using MATLAB (MathWorks, Natick, MA) for background subtraction, comparison of top, middle, and bottom intensity, and normalization, and plotted using Prism (GraphPad, La Jolla, CA).

Statistics and reproducibility. All fly crosses were repeated at least twice and ovary dissections and staining were repeated at least three times. The exact sample size $(n)$ is listed in Supplementary Data 1, representing biological replicates. Sample size was not predetermined by statistical methods but we used prior knowledge to estimate minimal sample size. The experiments were not randomized. Investigators were not blinded except when counting the $c 306-$ Gal4 fused egg chamber phenotypes.

Standard statistic tests were performed using Prism. Sample sizes were appropriately large with appropriate distributions. Unpaired $t$-test (two-tailed) was used for comparing two groups with similar variance as determined by $F$-test. Mann-Whitney nonparametric test (two-tailed) was used for comparing two groups with different variance. Ordinary one-way ANOVA, followed by Tukey's multiple comparisons test, was used for comparing multiple groups with similar variance as determined by Brown-Forsythe test. Kruskal-Wallis nonparametric test, followed by Dunn's multiple comparisons test, was used when the variance is significantly different among multiple groups. For box plots, the Tukey method was used for plotting whiskers and outliers.

Data availability. The authors declare that all data supporting the findings of this study are available within the article and its supplementary information files or from the corresponding author upon reasonable request.

Received: 9 December 2016 Accepted: 9 September 2017 Published online: 01 November 2017

\section{References}

1. Xie, T. \& Spradling, A. C. A niche maintaining germ line stem cells in the Drosophila ovary. Science 290, 328-330 (2000).

2. Song, X. \& Xie, T. DE-cadherin-mediated cell adhesion is essential for maintaining somatic stem cells in the Drosophila ovary. Proc. Natl Acad. Sci. USA 99, 14813-14818 (2002).

3. Mascré, G. et al. Distinct contribution of stem and progenitor cells to epidermal maintenance. Nature 489, 257-262 (2012).

4. Hsu, Y. C., Li, L. \& Fuchs, E. Transit-amplifying cells orchestrate stem cell activity and tissue regeneration. Cell 157, 935-949 (2014).

5. Losick, V. P., Morris, L. X., Fox, D. T. \& Spradling, A. Drosophila stem cell niches: a decade of discovery suggests a unified view of stem cell regulation. Dev. Cell 21, 159-171 (2011)

6. Spradling, A. C. in The Development of Drosophila melanogaster (ed. Bate, M.) 1-70 (Cold Spring Harbor Laboratory Press, 1993).

7. Margolis, J. \& Spradling, A. Identification and behavior of epithelial stem cells in the Drosophila ovary. Development 121, 3797-3807 (1995).

8. Nystul, T. \& Spradling, A. Regulation of epithelial stem cell replacement and follicle formation in the Drosophila ovary. Genetics 184, 503-515 (2010).

9. Torres, I. L., López-Schier, H. \& St Johnston, D. A Notch/Delta-dependent relay mechanism establishes anterior-posterior polarity in Drosophila. Dev. Cell 5, 547-558 (2003).

10. Larkin, M. K., Holder, K., Yost, C., Giniger, E. \& Ruohola-Baker, H. Expression of constitutively active Notch arrests follicle cells at a precursor stage during Drosophila oogenesis and disrupts the anterior-posterior axis of the oocyte. Development 122, 3639-3650 (1996).

11. Assa-Kunik, E., Torres, I. L., Schejter, E. D., Johnston, D. S. \& Shilo, B. Z. Drosophila follicle cells are patterned by multiple levels of Notch signaling and antagonism between the Notch and JAK/STAT pathways. Development 134, 1161-1169 (2007).

12. Shyu, L.-F., Sun, J., Chung, H.-M., Huang, Y.-C. \& Deng, W.-M. Notch Signaling and Developmental Cell-Cycle Arrest in Drosophila Polar Follicle Cells. Mol. Biol. Cell 20, 5064-5073 (2009).

13. Sahai-Hernandez, P. \& Nystul, T. G. A dynamic population of stromal cells contributes to the follicle stem cell niche in the Drosophila ovary. Development 140, 4490-4498 (2013).

14. Nystul, T. \& Spradling, A. An epithelial niche in the Drosophila ovary undergoes long-range stem cell replacement. Cell Stem Cell 1, 277-285 (2007).

15. Song, X. \& Xie, T. Wingless signaling regulates the maintenance of ovarian somatic stem cells in Drosophila. Development 130, 3259-3268 (2003).

16. Zhang, Y. \& Kalderon, D. Hedgehog acts as a somatic stem cell factor in the Drosophila ovary. Nature. 410, 599-604 (2001).

17. Castanieto, A., Johnston, M. J. \& Nystul, T. G. EGFR signaling promotes selfrenewal through the establishment of cell polarity in Drosophila follicle stem cells. eLife 3, e04437 (2014).

18. Kirilly, D., Spana, E. P., Perrimon, N., Padgett, R. W. \& Xie, T. BMP signaling is required for controlling somatic stem cell self-renewal in the Drosophila ovary. Dev. Cell 9, 651-662 (2005).

19. Wang, S. et al. Wnt signaling-mediated redox regulation maintains the germ line stem cell differentiation niche. elife 4, e08174 (2015).

20. Luo, L., Wang, H., Fan, C., Liu, S. \& Cai, Y. Wnt ligands regulate Tkv expression to constrain Dpp activity in the Drosophila ovarian stem cell niche. J. Cell Biol. 209, 595-608 (2015).

21. Zhang, Y. \& Kalderon, D. Regulation of cell proliferation and patterning in Drosophila oogenesis by Hedgehog signaling. Development 127, 2165-2176 (2000).

22. Silver, D. L. \& Montell, D. J. Paracrine signaling through the JAK/STAT pathway activates invasive behavior of ovarian epithelial cells in Drosophila. Cell 107, 831-841 (2001).

23. Bai, J. \& Montell, D. Eyes absent, a key repressor of polar cell fate during Drosophila oogenesis. Development 129, 5377-5388 (2002).

24. Liu, Y. \& Montell, D. J. Identification of mutations that cause cell migration defects in mosaic clones. Development 126, 1869-1878 (1999).

25. Forbes, A. J., Spradling, A. C., Ingham, P. W. \& Lin, H. The role of segment polarity genes during early oogenesis in Drosophila. Development 122, 3283-3294 (1996).

26. Tworoger, M., Larkin, M. K., Bryant, Z. \& Ruohola-Baker, H. Mosaic analysis in the drosophila ovary reveals a common hedgehog-inducible precursor stage for stalk and polar cells. Genetics 151, 739-748 (1999).

27. Chang, Y. C., Jang, A. C., Lin, C. H. \& Montell, D. J. Castor is required for Hedgehog-dependent cell-fate specification and follicle stem cell maintenance in Drosophila oogenesis. Proc. Natl Acad. Sci. USA 110, E1734-E1742 (2013).

28. DiNardo, S., Heemskerk, J., Dougan, S. \& O'Farrell, P. H. The making of a maggot: patterning the Drosophila embryonic epidermis. Curr. Opin. Genet. Dev. 4, 529-534 (1994). 
29. Marcelle, C., Stark, M. R. \& Bronner-Fraser, M. Coordinate actions of BMPs, Wnts, Shh and noggin mediate patterning of the dorsal somite. Development 124, 3955-3963 (1997).

30. Ouspenskaia, T., Matos, I., Mertz, A. F., Fiore, V. F. \& Fuchs, E. WNT-SHH antagonism specifies and expands stem cells prior to niche formation. Cell 164, 156-169 (2016).

31. Danesin, C. et al. Integration of telencephalic Wnt and hedgehog signaling center activities by Foxg1. Dev. Cell. 16, 576-587 (2009).

32. Forbes, A. J., Lin, H., Ingham, P. W. \& Spradling, A. C. Hedgehog is required for the proliferation and specification of ovarian somatic cells prior to egg chamber formation in Drosophila. Development 122, 1125-1135 (1996).

33. Olson, E. R. et al. Yan, an ETS-domain transcription factor, negatively modulates the Wingless pathway in the Drosophila eye. EMBO Rep. 12, 1047-1054 (2011).

34. Wang, X. \& Page-McCaw, A. A matrix metalloproteinase mediates longdistance attenuation of stem cell proliferation. J. Cell Biol. 206, 923-936 (2014).

35. Besse, F. \& Pret, A. M. Apoptosis-mediated cell death within the ovarian polar cell lineage of Drosophila melanogaster. Development 130, 1017-1027 (2003).

36. Tworoger, M., Larkin, M. K., Bryant, Z. \& Ruohola-Baker, H. Mosaic analysis in the drosophila ovary reveals a common hedgehog-inducible precursor stage for stalk and polar cells. Genetics 151, 739-748 (1999).

37. Borensztejn, A., Boissoneau, E., Fernandez, G., Agnès, F. \& Pret, A. M. JAK/ STAT autocontrol of ligand-producing cell number through apoptosis. Development 140, 195-204 (2013).

38. Sun, J. \& Deng, W. M. Hindsight mediates the role of notch in suppressing hedgehog signaling and cell proliferation. Dev. Cell 12, 431-442 (2007).

39. Housden, B. E., Millen, K. \& Bray, S. J. Drosophila reporter vectors compatible with $\Phi C 31$ integrase transgenesis techniques and their use to generate new notch reporter fly lines. G3 2, 79-82 (2012).

40. Ward, E. J. et al. Stem cells signal to the niche through the Notch pathway in the Drosophila ovary. Curr. Biol. 16, 2352-2358 (2006).

41. Song, X., Call, G. B., Kirilly, D. \& Xie, T. Notch signaling controls germline stem cell niche formation in the Drosophila ovary. Development 134, 1071-1080 (2007).

42. Grammont, M. \& Irvine, K. D. fringe and Notch specify polar cell fate during Drosophila oogenesis. Development 128, 2243-2253 (2001).

43. Vachias, C., Couderc, J. L. \& Grammont, M. A two-step Notch-dependant mechanism controls the selection of the polar cell pair in Drosophila oogenesis. Development 137, 2703-2711 (2010).

44. Grammont, M. \& Irvine, K. D. Organizer activity of the polar cells during Drosophila oogenesis. Development 129, 5131-5140 (2002).

45. López-Schier, H. \& St Johnston, D. Delta signaling from the germ line controls the proliferation and differentiation of the somatic follicle cells during Drosophila oogenesis. Genes Dev. 15, 1393-1405 (2001).

46. Vied, C., Reilein, A., Field, N. S. \& Kalderon, D. Regulation of stem cells by intersecting gradients of long-range niche signals. Dev. Cell 23, 836-848 (2012).

47. Reilein, A. et al. Alternative direct stem cell derivatives defined by stem cell location and graded Wnt signalling. Nat. Cell Biol. 19, 433-444 (2017).

48. Liu, Z. et al. Coordinated niche-associated signals promote germline homeostasis in the Drosophila ovary. J. Cell Biol. 211, 469-484 (2015).

49. $\mathrm{Li}, \mathrm{C}$. et al. Ci antagonizes Hippo signaling in the somatic cells of the ovary to drive germline stem cell differentiation. Cell Res. 25, 1152-1170 (2015).

50. Mottier-Pavie, V. I., Palacios, V., Eliazer, S., Scoggin, S. \& Buszczak, M. The Wnt pathway limits BMP signaling outside of the germline stem cell niche in Drosophila ovaries. Dev. Biol. 417, 50-62 (2016).

51. Upadhyay, M. et al. Transposon dysregulation modulates dwnt4 signaling to control germline stem cell differentiation in Drosophila. PLoS Genet. 12, e1005918 (2016)

52. Johnston, M. J., Bar-Cohen, S., Paroush, Z. \& Nystul, T. G. Phosphorylated Groucho delays differentiation in the follicle stem cell lineage by providing a molecular memory of EGFR signaling in the niche. Development 143, 4631-4642 (2016)
53. Hsu, Y. C., Li, L. \& Fuchs, E. Emerging interactions between skin stem cells and their niches. Nat. Med. 20, 847-856 (2014).

54. Tan, D. W. \& Barker, N. Intestinal stem cells and their defining niche. Curr. Top. Dev. Biol. 107, 77-107 (2014).

55. Campbell, C., Risueno, R. M., Salati, S., Guezguez, B. \& Bhatia, M. Signal control of hematopoietic stem cell fate: Wnt, Notch, and Hedgehog as the usual suspects. Curr. Opin. Hematol. 15, 319-325 (2008).

56. Takebe, N. et al. Targeting Notch, Hedgehog, and Wnt pathways in cancer stem cells: clinical update. Nat. Rev. Clin. Oncol. 12, 445-464 (2015).

57. Haack, T., Bergstralh, D. T. \& St Johnston, D. Damage to the Drosophila follicle cell epithelium produces "false clones" with apparent polarity phenotypes. Biol. Open 2, 1313-1320 (2013)

58. Kambadur, R. et al. Regulation of POU genes by castor and hunchback establishes layered compartments in the Drosophila CNS. Genes Dev. 12, 246-260 (1998)

59. Schindelin, J. et al. Fiji: an open-source platform for biological-image analysis. Nat. Methods 9, 676-682 (2012).

\section{Acknowledgements}

This work was supported by NIH grant GM46425 to D.J.M. We thank Abby Padgett, Benjamin Cheng and Junjie Luo for technical assistance. We thank Dr, Ward F. Odenwald and the Developmental Studies Hybridoma Bank for providing antibodies and Drs. Yashi Ahmed, Erika Bach, Andreas Bergmann, Sarah Bray, Anna Jiang, Todd Nystul, Andrea Page-McCaw, Mark Peifer, Ilaria Rebay, the Bloomington Drosophila Stock Center, and the Vienna Drosophila Resource Center for providing fly stocks. We acknowledge the use of the NRI-MCDB Microscopy Facility and the Imaris computer workstation supported by the Office of The Director, National Institutes of Health of the NIH under Award \# S10OD010610.

\section{Author contributions}

W.D. and D.J.M. conceived of the project. W.D., A.P., T.K., and H.B. performed the experiments. W.D., A.P., and D.J.M. prepared the manuscript. All authors participated in the design of the experiments, the interpretation of the data, and the production of the final manuscript.

\section{Additional information}

Supplementary Information accompanies this paper at doi:10.1038/s41467-017-01322-9.

Competing interests: The authors declare no competing financial interests.

Reprints and permission information is available online at http://npg.nature.com/ reprintsandpermissions/

Publisher's note: Springer Nature remains neutral with regard to jurisdictional claims in published maps and institutional affiliations.

Open Access This article is licensed under a Creative Commons Attribution 4.0 International License, which permits use, sharing, adaptation, distribution and reproduction in any medium or format, as long as you give appropriate credit to the original author(s) and the source, provide a link to the Creative Commons license, and indicate if changes were made. The images or other third party material in this article are included in the article's Creative Commons license, unless indicated otherwise in a credit line to the material. If material is not included in the article's Creative Commons license and your intended use is not permitted by statutory regulation or exceeds the permitted use, you will need to obtain permission directly from the copyright holder. To view a copy of this license, visit http://creativecommons.org/ licenses/by/4.0/

(C) The Author(s) 2017 